\title{
L'ÉQUATION D'ÉTAT ET LE PORTEUR ÉLÉMENTAIRE DE MAGNÉTISME DU NICKEL
}

\author{
Par M. Lovis NÉEL. \\ Institut de Physique de Strasbourg.
}

\begin{abstract}
Sommaire. - Dans l'introduction, on passe en revue les interprétations principales de l'aimantation à saturation et de la constante de Curie du nickel et on montre la nécessité, pour arriver à une conclusion définitive, d'interpréter dans son ensemble l'équation d'état magnétique du nickel. On propose ensuite un modèle de substance ferromagnétique, avec la loi de Lenz comme point de départ, qui permet un calcul complet de l'influence des fluctuations du champ moléculaire. Les propriétés du modèle sont comparées à celles du nickel, notamment la susceptibilité initiale (dans l'état paramagnétique), l'aimantation spontanée, la chaleur spécifique, etc... On apporte ensuite au modèle proposé quelques corrections suggérées par la mécanique quantique qui permettent d'interpréter quantitativement la loi d'approche à la saturation et le terme d'aimantation parasite de IVeiss et Forrer. Dans une dernière partie, on rassemble les arguments en faveur de l'exactitude de la notion de fluctuations du champ moléculaire. L'ensemble de l'équation d'état magnétique du nickel suggère l'interprétation suivante qui s'adapte le mieux aux faits expérimentaux : les propriétés magnétiques du nickel sont dues à un nombre variable de porteurs de moment, possédant un spin résultant $S=\frac{1}{2}$. Ce nombre est une fonction de l'aimantation. A la saturation,

il y a 0,607 porteur par atome de nickel et aux aimantations faibles il y en a 0,867 .
\end{abstract}

\section{Introduction.}

1. Interprétation de la saturation absolue et de la constante de Curie. - Le nickel est la seule substance ferromagnétique dont on connaisse avec précision l'équation d'état magnétique dans un large domaine, grâce aux expériences de MM. Weiss et Forrer (1) (2). On possède aussi des données très précises sur ses anomalies de chaleur spécifique qui sont étroitement liées aux propriétés magnétiques (3). Cependant, ces renseignements n'ont presque pas servi à l'interprétation générale du ferromagnétisme, car l'explication des propriétés particulières du nickel pose desproblèmes difficiles, dont on a proposé de multiples solutions, mais dont aucune ne s'impose définitivement.

Depuis le travail de M. Weiss sur la théorie du ferromagnétisme (4), on considère un corps ferromagnétique comme un corps paramagnétique soumis à un champ magnétique égal à la somme du champ extérieur et du champ moléculaire proportionnel à l'aimantation. Deux constantes fondamentales du nickel sont, à basse température, la saturation absolue $\sigma_{01}=57, \mathfrak{5}$, à haute température la constante de Curie $C=0,0055$ : ces deux constantes sont rapportées au gramme. En prenant l'atome comme porteur de moment magnétique,on en déduit que l'atome-gramme de nickel possède 3 magnétons de Weiss $\left(^{*}\right)$ à basse température et 8 magnétons dans l'état paramagné tique, en utilisant la loi du paramagnétisme de Langevin (ङ) dans ce calcul. Ces deux moments sont, au millième près, des multiples entiers et simples du magnéton. Rappelons ici que les ex-

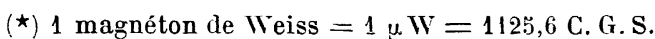

périences sur les sels paramagnétiques confirmentdans certains cas l'existence du magnéton, unité élémentaire. Les deux multiples 3 et 8 paraissent d'autant plus remarquables que leur différence est très voisine de 4,94, magnéton de Bohr. Le même système d'interprétation fournit une augmentation analogue du moment de 11 à $15,79 \mu \mathrm{W}$ pour le fer et de 9 à $1 \check{\partial} \mu \mathrm{W}$ pour le cobalt.

Malheureusement on aboutit ainsi à une impasse : l'hypothèse n'est susceptible d'aucune vérification expérimentale et l'équation d'état magnétique, pourtant si riche en détails, surtout au voisinage du point de Curie, reste en dehors de toute interprétation. D'ailleurs, l'étude théorique et expérimentale des sels des métaux rares montre que la formule de Langevin ne fournit en général qu'un moment apparent : il n'y aurait alors pas lieu de comparer les moments 3 et 8 .

Depuis longtemps, on a suggéré de prendre le spin de l'électron (6) comme porteur de moment magnétique. La saturation absolue donne 0,607 porteur par atome.

Comme je recherche ici, simplement, une interprétation formelle du ferromagnétisme, je n'essaierai pas de justifier l'existence d'un tel nombre de porteurs qui n'offre aucune relation simple avec le nombre des atomes, pas plus que pour le champ moléculaire, je n'aborde la question de son origine.

La loi d'aimantation paramagnétique est donnée par la formule de Lenz (7):

$$
\sigma=\sigma_{0}{ }^{\prime} \text { th } \frac{\mu_{0} H^{\prime}}{k T^{\prime}} .
$$

Si le nombre des porteurs de moment était le même 
à haute et à basse température, $\sigma^{\prime}$ serait égal à la saturation absolue $\sigma_{0}$; au contraire :

$$
\sigma_{0}^{\prime}=\frac{k C}{\mu .}=82,2
$$

c'est-à-dire 0,867 porteur par atome. De l'état ferromagnétique à l'état paramagnétique, le nombre des porteurs augmenterait de 43 pour 100 .

L'aimantation spontanée calculée dans cette hypothèse s'accorde beaucoup mieux avec l'expérience que celle qu'on calcule avec la formule de Langevin (8), mais des irrégularités subsistent au voisinage du point de Curie.

Dans les hypothèses précédentes, l'interprétation des phénomènes exige une augmentation du nombre des porteurs au point de Curie : on peut prétendre que cette augmentation n'est qu'une apparence due à l'emploi d'une loi d'aimantation incorrecte. C'est ainsi que Wolf $(y)$ pose que 3 atomes de nickel sur 10 sont dans l'état ${ }^{3} F$ et les 7 autres dans l'état $' D$. Cette hypothèse donne une saturation absolue correcte, mais le moment paramagnétique apparent calculé d'après ces données est de 7,6 au lieu de 8 magnétons, moment observé. Néanmoins, la possibilité d'une hypothèse de ce genre est à retenir.

2. Nécessité d'une interprétation d'ensemble de l'équation d'état magnétique. - La saturation absolue et la constante de Curie ne donnent ainsi que des renseignements très insuffisants pour conclure. L'étude d'ensemble de l'équation d'état magnétique s'impose. C'est au voisinage du point de Curie qu'on obtiendra les résultats les plus importants, car on peut y explorer une isotherme dans un large domaine d'aimantation, ce qui présente le même intérêt que l'étude d'un paramagnétique sans champ moléculaire au voisinage du zéro. Malheureusement, c'est dans cette région qu'on observe des phénomènes qui ne rentrent incontestablement pas dans le schéma simple de ferromagnétisme admis au début de cet article; c'est-à-dire paramagnétisme à champ moléculaire. Citons d'abord l'exislence d'un point de Curie paramagnétique différent du point de Curie ferromagnétique et, corrélativement, la courbure de plus en plus accentuée, à mesure que l'on s'approche du point de Curie, de la courbe $\left.\frac{1}{\%}=f(T){ }^{*}\right)$.

Ainsi, dans l'état paramagnétique, le moment apparent ${ }^{\star *}$ ) décroît à partir du point de Curie, ce qui est en désaccord, aussi bien avec les premières hypothèses d'une augmentation de moment qu'avec l'hypothèse d'un moment constant. Enfin, la courbe d'aimantation spontanée est beaucoup plus redressée au voisinage du point de Curie que ne le voudrait la théorie.

$\left.{ }^{\star}{ }^{\star}\right) \chi=$ susceptibilité rapportée an gramme.

$(\star \star)$ J'appelle moment apparent le moment calculé d'après la formule $: \mu=\sqrt{3 R . / C}$.
Outre l'intérêt intrinsèque de l'interprétation de ces phénomènes particuliers, leur explication préalable est indispensable pour tirer des isothermes magnétiques des renseignements d'ordre général sur la nature du porteur élémentaire de moment du nickel.

\section{Etude de l'influence des fluctuations sur un modèle semi-classique.}

3. Principes de la théorie des fluctuations du champ moléculaire. - Dans un travail antérieur (10), j'ai montré que les fluctuations du champ moléculaire créaient une différence entre les deux points de Curie, mais je n'avais tenté aucune application numérique. Les principales difficultés de théories analogues sont l'ordre mathématique : pour aboutir à des résultals numériques, il faut recourir à des modèles schématiques de substances magnétiques, mais les résultats ainsi obtenus n'en ont pas moins une portée générale.

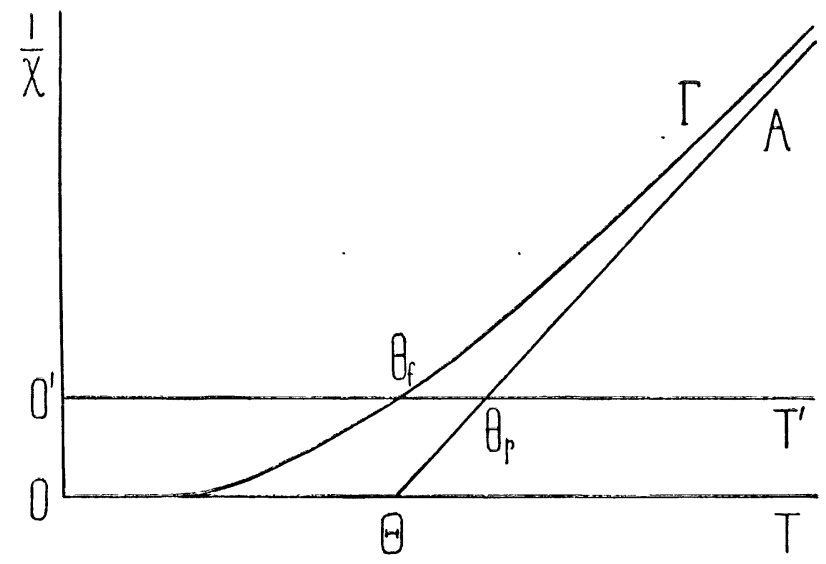

Fig. 1 .

Le principe d'explication que je propose est le suivant: je partage les actions mutuelles entre porteurs de moment magnétique en deux groupes. D'abord, les actions à longue distance dont les fluctuations dans le temps et l'espace sont négligeables et qu'il est légitime de remplacer par un champ moléculaire magnétique fictif, proportionnel à l'aimantation, et entièrement analogue au champ moléculaire de Weiss. Le deuxième groupe d'actions mutuelles comprendra les actions à courte distance qui fluctuent d'une manière importante : il faut les intégrer dès le début dans le calcul de la loi d'aimantation. Tout au moins dans le modèle adopté, ce dernier type d'interaction ne produit pas de ferromagnétisme,le modèle est encore paramagnétique, mais la loi classique du paramagnétisme $: \%=\frac{C}{T}$ est remplacée par une loi plus compliquée représentée schématiquement sur la figure 1 par la courbe $\Gamma$. Cette courbe tangente à l'origine à l'axe des températures est asymptote à une droite $\mathrm{A} \theta$. Introduisons maintenant 
les actions mutuelles à longue distance sous la forme d'un champ moléculaire :

$$
H_{m}=11 \mathrm{~s}
$$

le nouveau diagramme s'obtient (10, p. 40) en faisant subir à l'axe OT la translation : $00^{\prime}=u$. Le nouvel axe $0^{\prime} \mathrm{T}^{\prime}$ coupe la courbe en $\sigma_{f}$ et l'asymptote en $\theta_{p}$. Ces deux points sont respectivement les points de Curie ferromagnétique et paramagnétique. Si le champ moléculaire de ce même modèle était entièrement dépourvu de fluctuations, ${ }^{\circ}$. viendrait se confondre avec $\theta_{p}$ et $\frac{1}{\%}$ serait représenté par la droite $A \theta_{p}$. C'est donc $\theta_{p}$ qui est le véritable point de Curie, fonction du champ moléculaire total et indépendant des fluctuations. De même que la courbe réelle qui représente $\frac{1}{\%}$ en fonction de $T$, ne se détache qu'au voisinage lu point de Curie de la droite idéale $A \theta_{p}$, de mème la courbe réelle de l'aimantation spontanée derra se délacher progressivement de la courbe l'aimantation idéale, d’une substance sans fluctuations, aboutissant en $\theta_{P}$.

C'est effectivement ce que montre l'expérience. En admettant la loi d'aimantation 1), avec un champ moléculaire tel que le point de Curie soit à $378^{\circ}$, point de Curie paramagnétique du nickel, j’ai calculé l'aimantation spontanée en fonction de la température. J'ai représenté cette courbe sur la figure 2 . en traits interrompus ainsi que les données expérimentales de Weiss et Forrer, en trait plein. Le raccordement se fait d'une. manière inespérée.

Ce résultat justifie l'iłće d'attribuer aux fluctuations du champ moléculaire les anomalies de la région du point de Curie.

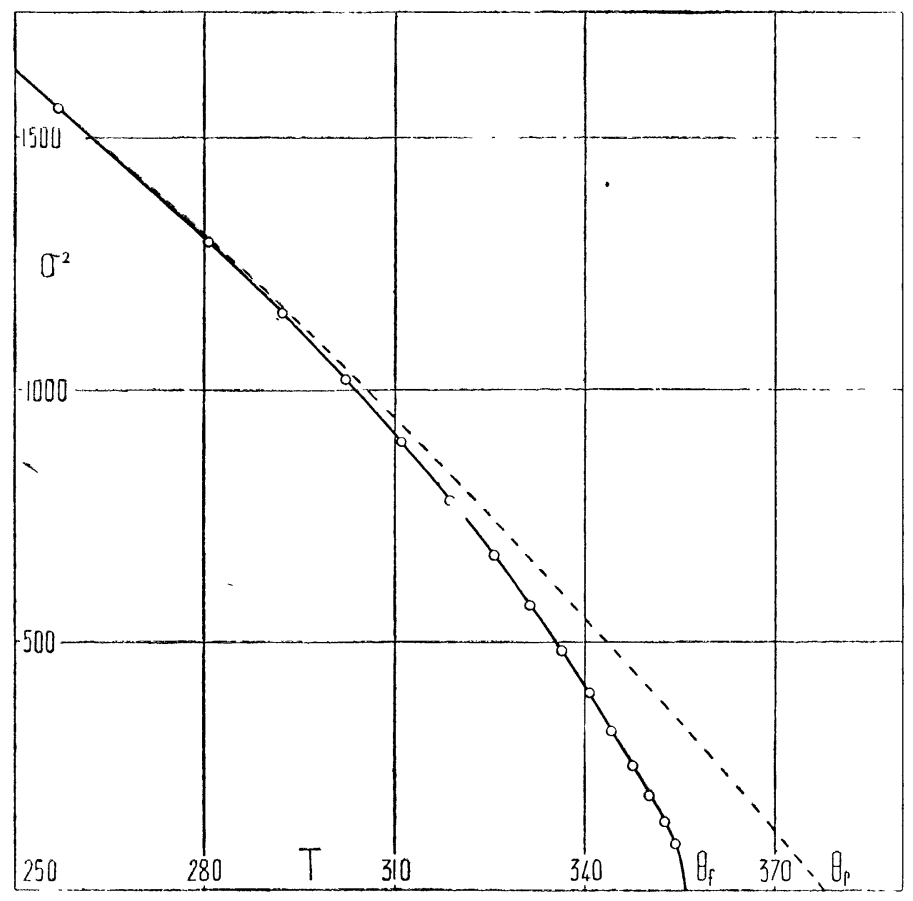

Fig. 2:

4. Modele de substance ferromagnétique et calcul. - Pour calculer l'effet des fluctuations, j'envisage la substance comme une collection de groupes. contenant chacun $\boldsymbol{N}$ porteurs de moment magnétique $\left(^{*}\right)$ : chacun des porteurs peut s'orienter soit dans le sens du champ magnétique soit en sens inverse. Chaque groupe possède $\frac{N !}{p ! q !}$ configurations distinctes dans lesquelles $p$ porteurs sont orientés dlans le sens ılu champ

( ) Dans les $\$ 16$ et suivants, je justifierai l'idée de conserver à $N$ une valeur finie et j’indiquerai les modifications que la nouvelle mécanique apporte au schéma semi-classique proposé ici. magnétique et $q$ en sens inverse. En l'absence l'actions mutuelles, 'e modèle est identique à celui de Lenz : il s'applique à des porteurs dont le spin résultant est égal à $S=\frac{1}{2}$. Je l'ái choisi, car il conduil à des calculs simples : de plus les courbes expérimentales d'aimantation spontanée se rapprochent beaucoup plus de celles qu'on obtient avec la loi de Lenz que de celles qu'on obtient avec la loi de Langevin.

On tiendra compte des actions mutuelles à courte distance, en introduisant une énergie potentielle entre porteurs. Soit donc $w$, l'énergie potentielle relative de deux porteurs quelconques antiparallèles pris à l'inté- 
rieur du groupe et 0 , l'énergie relative de deux porteurs quelconques parallèles. Vis-à-vis d'un porteur quelconque, tous les autres porteurs de son groupe jouent le même rôle. Entre deux groupes distincts, on supposera qu'il n'y a pas d'autres interactions que celles que l'on peut représenter au moỳen d'un champ moléculaire du type de Weiss.

Soit $\mu$ le moment magnétique d'un porteur, l'énergie potentielle d'un groupe $(p, q)\left(^{\star}\right)$ est :

$$
W=p q u-(p-q) p \cdot H
$$

$H$ est la somme du champ magnétique extérieur et du champ moléculaire.

D'après la formule de Boltzmann, la probabilité d'existence d'une configuration $(p, q)$ parmi toutes les configurations possibles s'écrit :

$$
\pi(p, q)=\frac{\frac{N !}{p ! q !} \mathbf{e}^{-\frac{W}{k T}}}{\sum \frac{N !}{p ! q !} \mathbf{e}^{-\frac{W}{k T}}}
$$

le signe $\Sigma$ est étendu à tous les entiers positifs assujettis à vérifier la relation : $p+q=N$.

Le moment magnétique d'une configuration $(p, q)$ est $(p-q)$ s. l'aimantation moyenne d'un groupe est donc : $\Sigma(p-q)$ p. $\varpi$. Pour un gramme, en désignant par $\sigma_{0}$ l'aimantation de toute la substance lorsque tous les porteurs sont alignés dans la direction du champ, l'aimantation $s$ s'écrit :

$$
\sigma=\sigma_{0} \sum \frac{p-q}{N} \pi
$$

Le nombre des porteurs d'un groupe joue le rồe d'un coefficient de fluctuation.Quand $N$ est petit'l'importance relative des fluctuations est grande.Au contraire quand $I$ tend vers l'infini,les propriétés du modèle se rapprochent de plus en plus de celles d'une substance où le champ moléculaire serait purement du type de Weiss.

Si jamais ce modèle donne une représentation convenable des fails expérimentaux, il ne faudra pas cepenlant en couclure que dans une substance magnétique réelle les porteurs s'assemblent par groupes de $N$ unités, ni que les actions muluelles entre un porteur et ses $I^{r}$ plus proches roisins soient équivalentes. Cet aspect schématique de la théorie n'est qu'un artifice de calcul et il faut se garder d'attribuer au coefficient. $Y$ sa signification littérale. Une infinité d'autres tỵpes d'actions mutuelles donneraient d'ailleurs des équations d'état magnétique présentant les mèmes caractéristiques générales. Je me propose simplement ici l'étudier les modifications que les fluctuations apportent à l'équạtion l'état: les lois réclles d'action mutuelle sont en dehors de mon sujet.

$\left.{ }^{\star}\right) p$ porteurs dans le sens du champ magnétique, q en sens inverse. i. Susceptibilité initiale (11). - Dans ce чui va suivre, je vais comparer avec l'expérience la formule (5), dans le cas du nickel. J'étudierai plus spécialement la susceptibilité initiale, l'aimantation spontanée et la chaleur spécifique. L'élude de la susceptibilité initiale est particulièrement précieuse pour déterminer les constantes. La formulr כ̃ , développée suivant les puissances croissantes le $\boldsymbol{H}$, śr.rit en s'arrêtant au terme en $H^{3}$ :

$$
\tau=\tau_{0}\left(a H-i a^{3} H^{3}\right)
$$

$a$ et $i$ ont les significations suivantes:

$$
a=\frac{A_{2} N \mu .}{A_{0} k T} \quad \lambda=\frac{A_{10^{2}}{ }^{2}}{A_{2}{ }^{2}}\left(\frac{A_{1}}{6.1}-\frac{A_{2}}{2 A_{11}}\right)
$$

en posant:

$$
A_{i}=\sum \frac{N^{\prime} !}{p ! q !} \frac{(\mu-q)^{i}}{N^{i}} \mathrm{e}^{-\frac{p \eta u^{\prime \prime}}{l^{\prime} l^{\prime}}}
$$

La susceptibilité initiale \%o est égale à $a \sigma_{0}$.

Nous allons rechercher l'asymptote à la courbe : $\frac{1}{\%}=f(T)$. Aux tempéralures élevées, l'exponentielle qui figure dans l'expression les $A_{i}$ se réduit à

$$
\left(1-\frac{p q u}{k T}\right)
$$

et un caleul simple donne :

$$
\frac{1}{\%}=\frac{1}{c_{0} \rho \cdot}\left[k T-\frac{w(N-1)}{2}\right] .
$$

Dans le système de coordonnées $\frac{1}{\%}, T$, c’est l'équation l'une droite qui coupe l'axe des $T$ au point :

$$
\Theta=\frac{w\left(N^{\prime}-1\right)}{2 h}
$$

c'est l'asymplote cherchée.

Ce résultat est conforme à celui que j’avais obtenu dans un travail antérieur $(10, \mathrm{p} .32): \frac{w\left(. \mathrm{N}^{2}-1\right)}{2}$ représente, en effel, l'ánergie moyenne do liaison par porteur.

Pour tenir comple du champ moléculaire à longue distance : $\left\|_{m}=\right\| \sigma$, il sufitira le relrancher $\|$ aux valeurs de $\frac{1}{\% .11}$ et finalement:

$$
\frac{1}{\%}=\frac{l_{1}}{\sigma_{0} ; s_{1} T} \frac{A_{0} T}{I_{2} T}-n
$$

c'est une fonction de la tempírature et des quatre constantes :

$$
\frac{\sigma_{0} \mu}{k}, \quad \theta, \quad \| \text { et } \quad I
$$

(i. Détermination des constantes. - A partir 
de 750 " $\mathrm{K}$, l'expérience montre que $\frac{1}{\%}$ est une fonction linéaire de la température, c’est-à-dire que la courbe se confond pratiquement avec son asymptote. Donc, en désignant par $C$ la constante de Curie expérimentale, on a

$$
\frac{\sigma_{0} \mu .}{k}=C
$$

Pour óliminer la constante $n$ et liminuer les tàlonnements, il est avantageux de comparer les courbes dérivées, $\frac{\partial \frac{1}{\%}}{\partial T}$ en fonction de $T$.

On choisira une valeur de $N$ et on calculera entièrement la courbe dérivée correspondante. On déterminera $\Theta$ de manière à la faire coïncider avec la courbe expérimentale. Si l'accord des deux courbes n'est pas satisfaisant on calculera la courbe dérivée pour une autre valeur de $\Lambda$.Je donne dans le tableau I, les valeurs

$$
\partial \frac{1}{4}
$$

de $\frac{\bar{\gamma}}{\partial T}$ en fonction de $T$, tirées des expériences de Weiss

et Forrer (colonnes 1 et 2 ), qui seules rassemblent d'une façon homogène les données para et ferromagné. tiques. Sur la figure 3, jai représenté les courbes théoriques :

$$
\frac{\sigma_{0} \mu .}{k} \frac{\partial}{\partial T} \frac{1}{\%}=f\left(\frac{T}{\Theta}\right)
$$

calculées pour les quatre valeurs : $N^{\top}=200 ; 400 ; 800$ et $1600\left(^{\circ}\right)$.

En admettant les valeurs provisoires $\Theta=613^{\circ}, t^{\circ}$ et ( $=0,00536$, j'ai calculé les valeurs réduites expérimentales $C \frac{\partial}{\partial T} \frac{1}{\chi}$ en fonction de $\frac{T}{\Theta}$ (colonnes 3 et 4 du tableau I) qui sont directement comparables aux valeurs théoriques d'après la relation $(10)$.

Elles sont représentées par des cercles sur la figure 3 . Ces points sont très voisins de la courbe $N=800$. Un calcul ultérieur montre qu'avec $\Lambda^{r}=7$ ö) les écarts sont de l'ordre de grandeur des erreurs d'expérience. Dans le tableau II, je lonne pour $\Lambda^{\prime}=7$ ö0 les valeurs de $\frac{\dot{\sigma}_{0} \mu}{k} \frac{\partial \frac{1}{\%}}{\partial T}$ en fonction de $\frac{T}{\Theta}$.

(*) J'ai commencé par calculer les valeurs de $\frac{1}{\%}$ : puis j'ai dérivé graphiquement par rapport à $T$. Le calcul de $\frac{1}{\%}$ nécessite celui des quantités $A_{\text {u }}$ et $A$, qui sont des sommes étendues à un très grand nombre de termes, 200 au minimum. Avec une très grande approximation on peul les remplacer par des intégrales définies calculables par la méthode de Simpson. Il suffit de diviser l'intervalle d'intégration en une dizaine de parties pour avoir une précision relative de un millième. Les calculs ne présentent na-

\begin{tabular}{|c|c|c|c|}
\hline$T$ & $\frac{\partial \frac{1}{4}}{\partial T}$ & $\frac{T}{\theta}$ & $c \frac{\partial \frac{1}{\%}}{\partial T}$ \\
\hline 637.5 & 105,1 & 0,490 & 0,560 \\
\hline 640,3 & 109,4 & $0,99 \check{~}$ & 0,588 \\
\hline 643.9 & 115,7 & 1,000 & 0,622 \\
\hline 647,6 & 121,8 & 1,006 & 0,655 \\
\hline $6 \ddot{1,5}$ & 127,2 & 1,012 & 0,684 \\
\hline 650 & 133,1 & 1,018 & 0,716 \\
\hline 662,3 & 141.3 & 1,029 & 0.760 \\
\hline 673,2 & 152,6 & 1,046 & 0.820 \\
\hline 683,0 & 161,9 & 1,064 & 0.870 \\
\hline 697,5 & 165,7 & 1,084 & 0,891 \\
\hline 709,2 & 170,9 & 1,102 & 0.918 \\
\hline 726,7 & 177,6 & 1.129 & 0.953 \\
\hline 784 , & 181,9 & 1.163 & 0.978 \\
\hline 774,2 & 186.9 & 1.203 & 1,005 \\
\hline 820,2 & 186,0 & 1.274 & 1.000 \\
\hline 851,0 & 185,3 & 1.327 & 0,996 \\
\hline
\end{tabular}
turellement pas d'autre tifficulté que leur longueur.
Tableat I.

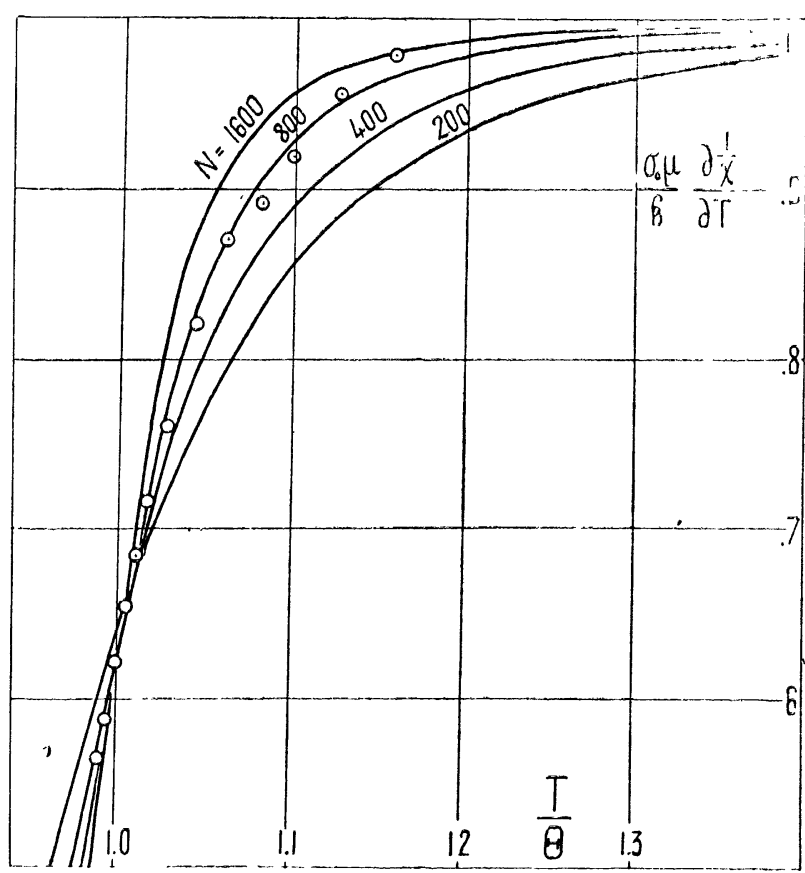

Fig. 3 .

Les comparaisons précédentes fournissent une valeur corrrecte de $I^{\prime}$ et une valeur approchée de $\Theta$. La dernière constante " $"$, s'obtiendra en comparant directement les valeurs calculées aux valeurs expérimentales de la susceptibilité. Si besoin est, on retouchera les valeurs admises pour $\Theta$ et pour $\frac{\sigma_{0} \mu}{k}$. 
Tableau II $(N=750)$.

\begin{tabular}{|c|c||c|c|}
\hline$\frac{T}{\Theta}$ & $\frac{\sigma_{0} \mu}{k} \frac{\partial \frac{1}{\gamma}}{\partial T}$ & $\frac{T}{\Theta}$ & $\frac{\sigma_{0} \mu}{k} \frac{\partial \frac{1}{\gamma}}{\partial T}$ \\
\cline { 1 - 2 } & & & \\
\hline 1,6037 & 1,000 & 1,0064 & 0,647 \\
1,4855 & 0,996 & 0,9988 & 0.603 \\
1,3828 & 0,993 & 0,9939 & 0,560 \\
1,2934 & 0,990 & 0,9890 & 0,527 \\
1,2148 & 0,978 & 0,9841 & 0,491 \\
1,1453 & 0,954 & 0,9793 & 0,455 \\
1,0976 & 0,917 & 0.9745 & 0,416 \\
1,0683 & 0,874 & 0,9698 & 0,379 \\
1.0458 & 0,820 & 0,9651 & 0,341 \\
1,0297 & 0,765 & 0,9605 & 0,307 \\
1,0166 & 0,701 & 0.9559 & 0,272 \\
\hline
\end{tabular}

Les valeurs définitivement adoptées sont les suivantes :

$N=730 \quad \Theta=642^{\circ} 8 \quad n=2400 \frac{k \Theta}{s_{0} \mu}=120000$.

La signification de $\frac{k \Theta}{\sigma_{0} \mu}$ est évidente : c'est le coefficient du champ moléculaire de Weiss équivalent, les fluctuations étant négligées, aux actions mutuelles à courte distance. On voit ainsi qu'au point de vue énergétique, les actions à longue distance ne représentent que le cinquantième des actions à courte distance :

$$
n=0,02 \frac{k \Theta}{\sigma_{0} \mu} \text {. }
$$

7. La théorie et l'expérience. - Dans le tableau III, je donne, à différentes températures (colonne 1), les valeurs calculées $\frac{1}{\chi}$ avec les coefficients de la formule $(11)$ (colonne 2). Dans la colonne 3, j'ai inscrit les valeurs expérimentales de $\frac{1}{\%}$, correspondant aux mêmes températures, interpolées d'après les expériences de Weiss et Forrer.

J'ai choisi ce procédé de comparaison parce que les auteurs ont fait un très grand nombre de déterminations : les interpolations sont ainsi faciles et sûres. Les valeurs indiquées ont été ramenées au champ 0 et corrigées du champ démagnétisant. Enfin, dans la colonne 4. je donne les différences relatives en pour 100 entre les valeur's calculées et les valeurs observées. Les deux courbes s'accordent, à 1 pour 100 près, dans tout l'intervalle. Les différences tantòt négatives, tantòt positives, varient assez irrégulièrement. Il est possible qu'un ajustement plus minutieux des constantes $-N$ en particulier - améliore l'accord, mais le résultat serait hors de proportion avec les longs calculs qu'il nécessiterait : d'une part, le modèle utilisé est. certainement trop éloigné de la réalité pour qu'un accord au millième près soit vraiment significatif; d'autre part, aux hautes températures, les expériences de Weiss et Forrer sont un peu moins précises quau voisinage du point de Curie, la méthode se prêtant difficilement aux mesures paramagnétiques. En effet, le moment apparent ainsi calculé est de $\overline{7}, 9 \mu \mathrm{W}$, alors que les mesures paramagnétiques fournissent d'ordinaire $8 \mu . W$. Cependant, dans la suite, pour discuter sur un ensemble homogène d'expériences, je prendrai toujours $C=0,0053$ s̆, valeur de Weiss et Forrer.

TABLBAU III.

\begin{tabular}{|c|c|c|c|}
\hline$T$ & $\frac{1}{\chi}$ calc. & $\frac{1}{\chi}$ obs. & dilf. p. 100 \\
\hline $919^{\circ} 5$ & 49900 & วั0 208 & $-0,6$ \\
\hline $858^{\circ} 2$ & 38530 & 38763 & $-0,6$ \\
\hline $804^{\circ} 6$ & 28622 & 28883 & $-0,9$ \\
\hline $7 \check{7} 7^{\circ} 23$ & 19990 & 19963 & $+0,1$ \\
\hline $715^{\circ} 17$ & 12290 & 12423 & $-1,0$ \\
\hline $695^{\circ} 84$ & 9184 & $911 \%$ & $+0,7$ \\
\hline $677^{\circ} \mathrm{\jmath} 3$ & $\begin{array}{ll}6 & 197\end{array}$ & $\begin{array}{ll}6 \quad 172\end{array}$ & $+0,4$ \\
\hline $667^{\circ} 00$ & 4586 & 4572 & $+0,3$ \\
\hline 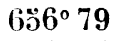 & $\begin{array}{ll}3 & 129\end{array}$ & 3134 & $-0,2$ \\
\hline $630^{\circ} 15$ & 2260 & 2273 & $-0,6$ \\
\hline $643^{\circ} 65$ & 1475 & 1491 & $-1,1$ \\
\hline $640^{\circ} 45$ & 1114 & 1132 & $-1, \mathfrak{0}$ \\
\hline $637^{\circ} 28$ & 783 & 789 & $-0,7$ \\
\hline $634^{\circ} 14$ & 474 & 468 & $+1,2$ \\
\hline
\end{tabular}

L'accord entre la théorie et l'expérience est surtout satisfaisant si l'on remarque que, dans l'intervalle de température exploré, la susceptibilité varie dans le rapport de 1 à 265 . La courbe théorique est tracée sur la figure 4, les cercles représentent les points expérimentaux. A l'échelle adoptée, l'accord est complet.

Le point de Curie paramagnétique $\theta_{p}$, d'après les constantes déterminées plus haut, esı à $\frac{T^{p}}{\Theta}=1,02000$ soil 382" 45, tandis que l'expérience donne $378^{\circ}$. La courbe, quoique parallèle à son as ymptote, en est encore à une certaine distance.

Par interpolation, le point de Curie ferromagné-

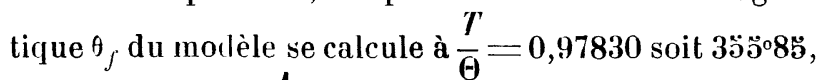
la valeur de $\frac{\sigma_{0} \mu}{k} \frac{\partial \frac{1}{\psi}}{\partial T}$ correspondante est 0,446 . La différence des deux points de Curie est : $\sigma_{p}-\theta_{f}=\mathbf{2} 6^{\circ} 6$.

Dès maintenant, il apparaît que l'introduction des fluctuations fournit la clé des anomalies observées dans la région du point de Curie : dautre part, les cons. tantes caractéristiques du champ moléculaire, $\Theta, N$ et $n$ ont été déterminées avec précision. Nous allons montrer, maintenant, qu'une comparaison plus poussée entre la théorie et l'expérience fournit encore des résutats cohérents. 


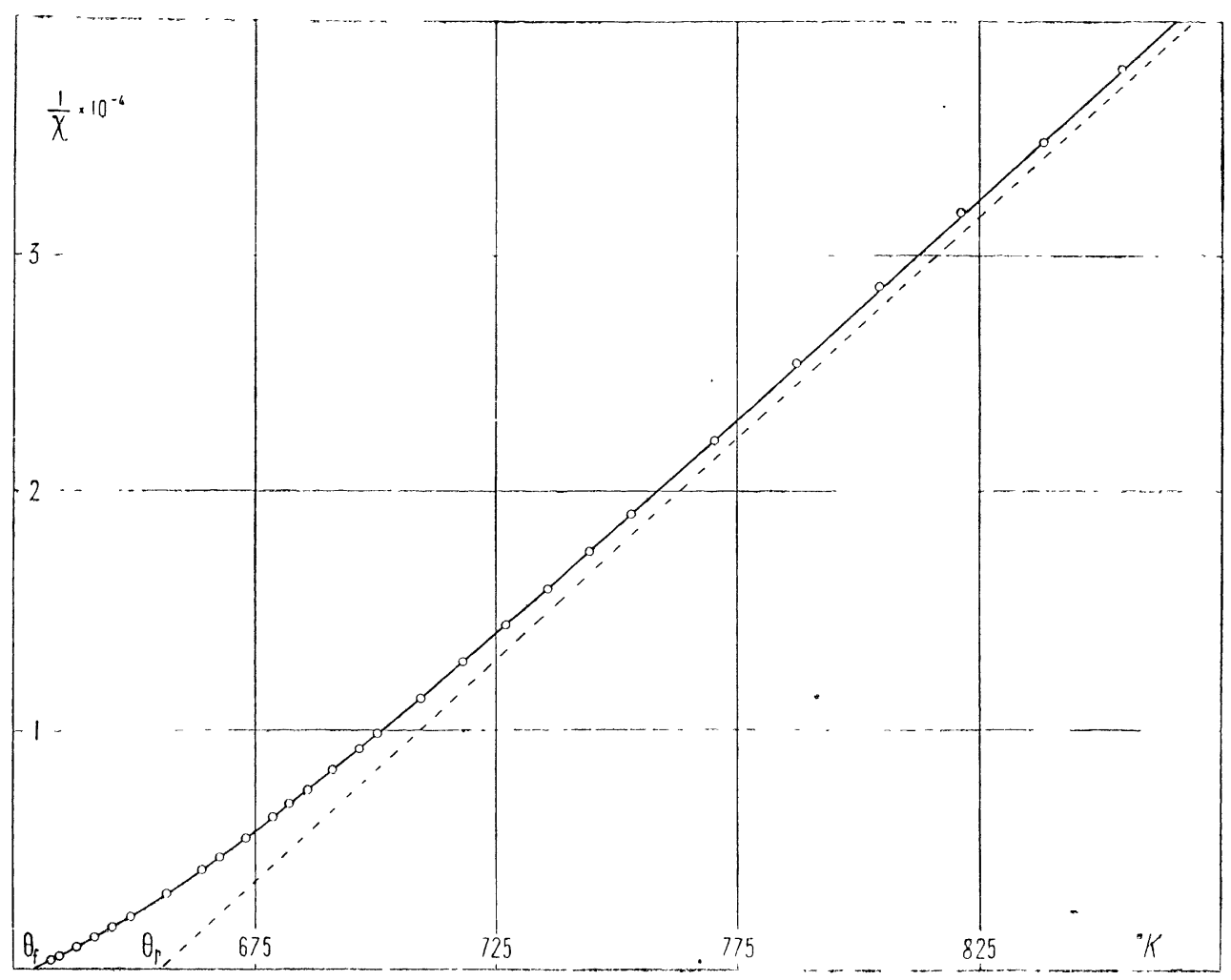

Fig. 4.

8. Calcul du deuxième terme de la loi d'aimantation. - La loi d'aimantation d'une substance paramagnétique quelconque s'écrit :

$$
\sigma=\alpha^{\prime} \boldsymbol{H}+\beta^{\prime} \boldsymbol{H}^{3}+\ldots
$$

$\boldsymbol{H}$ désigne le champ exlérieur : $x^{\prime}, \beta^{\prime}$ sont des fonctions de la température. En général, le ler'me en $H^{3}$ n'est pas accessible expérimentalement. Lorsque la substance suit la loi de Curie, on peut cependant le délerminer en expérimentant au voisinage du zéro absolu. Lorsqu'elle possède un point de Curie, c'est au voisinage de celui-ci que le coefficient $\beta^{\prime}$ est calculable au moyen des résultats expérimentaux.

Dans le modèle que j'ai adopté, pour calculer les coefficients $\alpha^{\prime}, \beta^{\prime}$, du développemen (13), il faut prendre la loi d'aimantation paramagnétique qui tient compte des actions mutuelles à courte distance et que j'écris pour abréger :

$$
\sigma=x \boldsymbol{H}^{\prime}+\beta \boldsymbol{H}^{\prime 3}+\ldots
$$

$H^{\prime}$ représente le champ interne total: $H^{\prime}=H+H_{m}$. On a donc :

$$
\sigma=\alpha(\boldsymbol{H}+\| s)+\beta(\boldsymbol{H}+11 \sigma)^{j}+\ldots
$$

En remplaçant dans cette équation $\sigma$ par sa valeur tirée de l'équation 13), et en identifiant dans les deux membres les coefficients de $\boldsymbol{H}$ et de $H^{i}$, j'ai :

$$
\alpha^{\prime}=\frac{\alpha}{1-n \alpha} \quad \beta^{\prime}=\beta \frac{\alpha^{\prime 4}}{\alpha^{4}} .
$$

Dès à présent, remarquons le grand avantage qu'il y a à opérer près du point de Curie : le rapport $\frac{\beta^{\prime}}{\alpha^{\prime}}$ des deux coefficients du développement (13) s'écrit en effet :

$$
\frac{\beta^{\prime}}{\alpha^{\prime}}=\frac{\beta}{\alpha} \frac{\alpha^{\prime 3}}{\alpha^{3}}
$$

c'est-à-dire quilil est égal au rapport $\frac{\beta}{\alpha}$ des coefficients d'une substance purement paramagnétique, multiplié par le rapport $\left(\frac{\alpha^{\prime}}{\alpha}\right)^{3}$ qui devient très grand au point de Curie : en négligeant les fluctuations on a, en effet :

$$
\frac{\alpha^{\prime}}{x}=\frac{T}{T-\theta}
$$

Pratiquement, ceci signifie que la susceptibilité varie avec le champ magnétique à partir d'une aimantation l'autant plus faible qu'on est plus près du point de Curie.

9. Calcul de $\frac{\partial \frac{1}{\%}}{\partial H^{2}}$. Pour dégager des résultats 
expérimentaux le coefficient $\beta^{\prime}$, il est avantageux de représenter graphiquement $\frac{1}{\%}$ en fonction du carré du champ magnétique. Un oblient une droite dont l'intersection avec l'axe des ordonnées fournit $\frac{1}{\alpha^{\prime}}$. La pente de cette droite est :

$$
\frac{\partial \frac{1}{\varkappa}}{\partial H^{2}}=-\frac{\beta^{\prime}}{\alpha^{\prime 2}}=-\beta \frac{\alpha^{\prime 2}}{\alpha^{4}}
$$

mais par définition d'après l'équation (6) :

$$
x=\sigma_{0} a \quad \beta=-\sigma_{0} \lambda a^{j},
$$

d'où :

$$
\beta=-\sigma_{0} \frac{\lambda \alpha^{3}}{\sigma_{0}{ }^{3}}=-\frac{\lambda \alpha^{j}}{\sigma_{00^{2}}{ }^{2}} \text { et } \quad \frac{\partial \frac{1}{\gamma}}{\partial \boldsymbol{H}^{2}}=\frac{\lambda}{\sigma_{0}{ }^{2}} \frac{\alpha^{\prime 2}}{\alpha} \text {. }
$$

Le coefficient $\lambda$ de la formule $(1 \dot{5})$ est donné au moyen des quantilés $A_{0}, A_{2}$ el $1_{4}$ par la formule ( 7$)$.

C'est une fonction de $N$ et de $\frac{T}{\Theta}$. J'ai calculé pour $N=7 \ddot{0}$, les valeurs de $\lambda$ pour différentes valeurs de

\begin{tabular}{|c|c|c|c|}
\hline$\frac{T}{\Theta}$ & $\lambda$ & $\frac{T}{\Theta}$ & $\lambda$ \\
\hline 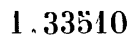 & 1,284 & 1.00133 & 3.336 \\
\hline 1. 20166 & 1. 440 & 0.99633 & 3.310 \\
\hline 1. 17803 & 1,890 & 0.99142 & 3.248 \\
\hline 1,11939 & 2.409 & 0,98653 & 3,161 \\
\hline 1.08252 & 2,768 & 0.98169 & 3.063 \\
\hline $1,03 ั 403$ & 3.126 & 0.97691 & ‥ 932 \\
\hline 1,03765 & 3.297 & 0,97216 & $2.8 \approx 9$ \\
\hline 1,02177 & 3,410 & 0.96281 & $2, \breve{56 \prime}$ \\
\hline 1.01144 & 3,413 & 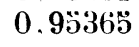 & $2,29 \prime$ \\
\hline
\end{tabular}
$\frac{T}{\Theta}$.

TABleau IV.

Elles sont rassemblées dans le tableau 1V. D'autre part $\alpha^{\prime}$, susceptibilité dans un champ infiniment faible, est connue par extrapolation de la courbe $\frac{1}{\%}=f\left(H^{2}\right)$. $\alpha$ se calcule par la relation : $\frac{1}{\alpha}=\frac{1}{x^{\prime}}+n$. Donc, la formule 15) permet finalement de calculer $\dot{\sim}_{0}$ en fonction de $\frac{\partial \frac{1}{\%}}{\partial \boldsymbol{H}^{\prime}}$.

10. Valeurs expérimentales de $\frac{\partial \frac{1}{\%}}{\partial H^{2}}$. Wans les expériences de Weiss et Forrer, 4 isothermes se prètent à une détermination assez précise de $\frac{\partial \frac{1}{\%}}{\partial H^{2}}$ : elles correspondent aux lempératures centigrades suivantes : $376^{\circ} 4 \overline{7} ; 380^{\circ} 15 ; 383^{\circ} 83$ et $387^{\circ} 50$. Les isothermes correspondant à des températures inférieures ne conviennent pas, car, dans les champs magnétiques utilisés, $\frac{1}{\chi}$ 'est plus une fonction linéaire de $\boldsymbol{H}^{2}$ : il faut, dans la formule de l'aimantation, tenir compte des termes en $H^{\text {s }}$ et des suivants. D’autre part, aux lempératures supérieures à $387^{\circ}$ ö 0 , la détermination de $\frac{\partial \frac{1}{\chi}}{\partial H^{2}}$ n'offre plus aucune précision. Déjà, à $387^{\circ} 0$, la précision relative n'est que de $\mathbf{1 0}$ pour $\mathbf{1 0 0}$. Dans les trois premières colonnes du tableau $v$, je donne les tempéra-

tures des isothermes avec les valeurs de $\frac{1}{\alpha^{\prime}}$ el de $\frac{\partial \frac{1}{\%}}{\partial H^{2}}$ correspondantes. La colonme 4 donne la précision rela-

\begin{tabular}{|c|c|c|c|c|c|c|}
\hline$T$ & $\frac{1}{\alpha^{\prime}}$ & $\frac{\partial \frac{1}{\%}}{\partial H^{2}}$ & p. 100 & $\sigma_{0}$ & $H_{\text {sup. }}$ & $\begin{array}{c}\text { Osup. } \\
7\end{array}$ \\
\hline $\begin{array}{l}369^{\circ} 07 \\
372^{\circ} 77\end{array}$ & & & & $\begin{array}{l}57.0 \\
58,0\end{array}$ & $\begin{array}{ll}21 & 313 \\
21 & 315\end{array}$ & $\begin{array}{c}11,2 \\
9.68\end{array}$ \\
\hline $376^{\circ} 47$ & 2162 & 0,820 & 2 & $63 . \ddot{3}$ & 17775 & 7,21 \\
\hline $380^{\circ} 15$ & 2661 & 0.5844 & 2 & 64.6 & 21315 & 7.21 \\
\hline $383^{\circ} 83$ & 3133 & 0.428 & 4 & 67.0 & $\mid 21313$ & 6.34 \\
\hline $387^{\circ} 50$ & 3654 & $0.31 \mathrm{~s}$ & 10 & 69.5 & $17 \quad 775$ & 4,68 \\
\hline $358^{\circ} 90$ & 253.8 & 24.6 & 2 & 71,9 & 758 & 2.8 \\
\hline
\end{tabular}
tive de la détermination de $\frac{\partial \frac{1}{\gamma}}{\partial H^{2}}$ en pour 100.

\section{T.ibleau V.}

A ces données, j'ai ajouté un résultat extrait d'une série de mesures que j'ai faites et qui seront prochainement publiées, sur l'aimantation du nickel au voisinage du point de Curie, dans des champs magnétiques de 100 à 1000 gauss. Cette mesure a donné lès résultats suivants :

\begin{tabular}{|l|l|l|l|l|l|}
\hline $\begin{array}{l}H \text { gauss. } \\
1\end{array} \ldots . .5$ & 179.9 & 293.8 & 443.0 & 577.0 & 737.6 \\
\hline$\%$ & 255.7 & 238,7 & 261.8 & 268.0 \\
\hline
\end{tabular}


La température est restée conslante à $\frac{1}{200}$ degré près pendant la durée des mesures. En portant $\frac{1}{\%}$ en fonction de $H^{\lrcorner}$(fig. $\ddot{3}$ ), on obtient une excellente droite.

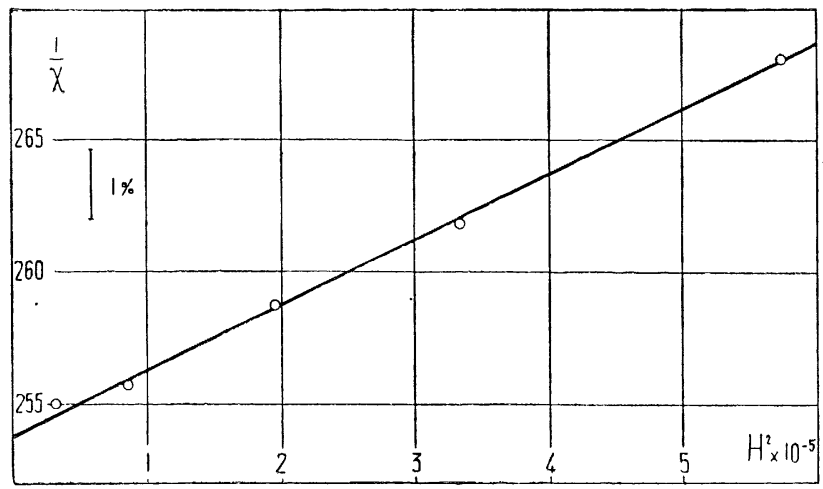

Fig. 3.

11. Étude des variations de $\sigma_{0}$. - J'ai donc finalement $\ddot{3}$ valeurs de $\frac{\partial \frac{1}{\psi}}{\partial \boldsymbol{H}^{2}}$ qui permettent de calculer les $\dddot{\jmath}$ valeurs de $\sigma_{0}$ reportées dans la colonne $\leftrightarrows$ du tableau $\mathrm{V}$. Remarquons que l'erreur relative sur $\sigma_{0}$. est la moitié de l'erreur relative sur $\frac{\partial \frac{1}{\gamma}}{\partial H^{2}}$.

Enfin, anticipant sur les résultats des paragraphes suivants, j'ai déterminé $\sigma_{0}$, par une autre méthode, aux températures de $372^{\prime \prime}, 77$ et de $369^{\circ}, 07$ : en comparant les isothermes complètes, théoriques et expérimentales.

Si le modèle de substance ferromagnétique proposé était entièrement satisfaisant, $\sigma_{0}$ serait constant et égal à la saturation absolue 57,5 . Il n'en est rien : $\sigma_{0}$ varie dans de larges limites. Les résultats précédents montrent aussi que $\sigma_{0}$ ne présente pas de relation apparente avec la température. On peut alors songer à une action du champ moléculaire. Pour être plus général, cherchons une relation entre $\sigma_{0}$ et l'aimantation. En dernière analyse, $\sigma_{0}$ est déterminé par la pente d'une droite tracéc sur un graphique qui contient en abscisse le carré du champ magnétique : c'est donc le point obtenu lans le champ magnétique le plus élevé qui joue le ròle principal dans le tracé de la droite. J'étudierai donc $\sigma_{0}$

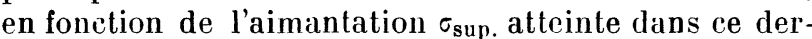
nier champ magnétique (colonne 6 et 7 du tableau 5 ). En reportant ces points sur un graphique (fig. 6), on constate une relation évidente entre $\sigma_{0}$ et $\sigma_{\text {sap. }}: \sigma_{0}$ diminue quand l'aimantation croìt. Ce phénomène est en accord avec l'hy pothèse d'une augmentation du moment ou du nombre des porteurs au point de Curie.

Puisque on est amené à conclure que $\sigma_{0}$ est une fonc- tion de l'aimantation, $\frac{1}{\%}$ n'est plus une fonction linéaire de $\boldsymbol{H}^{2}$, et les raisonnements précédents sont à modifier légèrement. Je ne les reprendrai pas pour ne pas allonger outre mesure cet exposé. J'ai simplement calculé, pour les isothermes étudiées, les valeurs de $\frac{1}{\gamma}$ en fonction de $H^{*}$, en admettant $\sigma_{0}$ correctement représenté en fonction de l'aimantation par la droite de la figure 6.

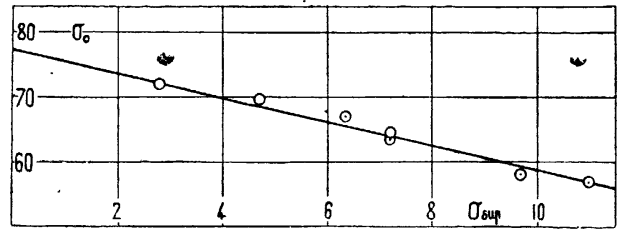

Fig. 6 .

Les courbes ainsi calculées ont été représentées, en trait plein, sur la figure 7 . Ce sont presque des droites sauf au voisinage de $H^{2}=0$.

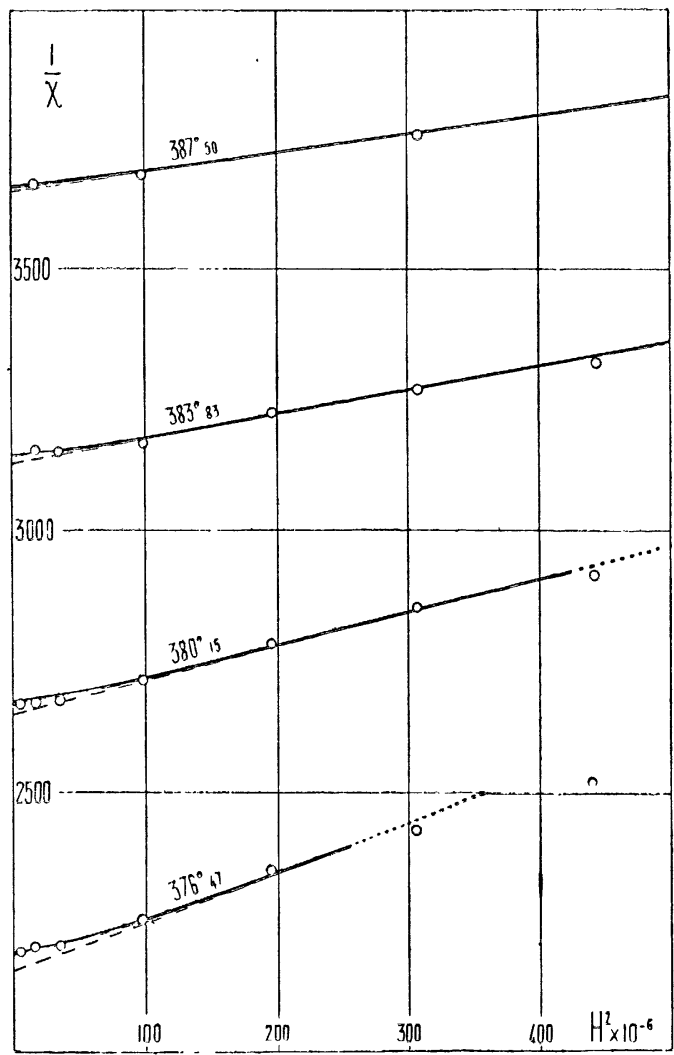

Fig. 7.

Cette petite correction améliore l'accord avec l'expérience. L'examen attentif des points expérimentaux montreainsi,en dehors de toute interprétation théorique, que la convexité de la courbe $\frac{1}{\%}=f\left(H^{2}\right)$ est dirigée 
vers l'axe des $H^{2}$, de sorte qu'on peut poser, pour des aimantations faibles et à une température donnée $T$ :

$$
\frac{1}{\%}=a+b H^{2}+c H^{i}+\ldots
$$

$a, b, c$, étant tous les trois positifs. Ce résultat sera utilisé dans la conclusion.

Pour $\sigma=0$, on a $\sigma_{0}=77 \pm 2$ (d'après la fig. 7 ). Mais la quantité $\sigma_{0}$ qui figure dans la constante de Curie $C=\frac{\sigma_{0} \mu}{k}=0,0053$ est également relative à des aimantations faibles, c'est donc la valeur $7 \bar{i}$ qu'il faut lui attribuer, d'où $\mu=5782$. Cette valeur n'est pas très éloignée de 5561 , magnéton de Bohr. L'expérience montre donc que la résultante des spins d'un porteur est égale à $\frac{1}{2}$.

Les faits précédents suggèrent la représentation suivante : dans $1 \mathrm{~g}$ de nickel, il y a un certain nombre de groupes de $N$ porteurs. Le nombre $N$ des porteurs d'un groupe sera toujours pris comme fixe et égal à 750 .

On considérera, au contraire, comme variable avec l'aimantation le nombre des groupes. Une première approximation des valeurs de l'aimantation spontanée et de la chaleur spécifique sera ainsi obtenue, en prenant comme nombre de groupes celui qui correspond à l'aimantation à saturation.

12. L’aimantation spontanée. - Le calcul de l'aimantation spontanée $\sigma_{\text {sp. peut se faire au moyen }}$ de la formule approchée (13), mais il n'est valable que pour des aimantations faibles - le dixième de la saturation au maximum - aussi est-il préférable d'utiliser la formule rigoureuse (ら) :

$$
\sigma=\sigma_{0} \sum \frac{p-q}{N} \omega
$$

La méthode consiste à déterminer par points une petite portion d'isotherme dans des champs magnétiques extérieurs faibles et à interpoler de manière à calculer l'aimantation $\sigma_{\mathrm{sp}}$. dans un champ extérieur nul.

La quantité $W$ est la seule inconnue qui rentre dans l'expression de $\varpi$, formule (4), puisque $N$ et $T$ sont donnés. Mais, d'après (3) :

$$
W=p q w-(p-q) \mu(\boldsymbol{H}+n \sigma)
$$

Les coordonnées d'un point d'une isotherme s'obtiennent en se donnant, a priori, une valeur $m$ de la variable auxiliaire $\mu(H+n \sigma)$. On peut alors calculer $W$, ๘ puis $\frac{\sigma}{\sigma_{0}}$ en fonction de $m$. Puis on a :

$$
\mu . H=m-\mu n \sigma=m-0,02 k \Theta \frac{\sigma}{\sigma_{0}},
$$

d'après la relation (12) du paragraphe 7 . En attribuant à $m$ d'autres valeurs, on calcule d'autres points et on peut tracer une isotherme en fonction des coordonnées $\frac{\sigma}{\sigma_{0}}$ et $\cdot . H$ : l'intersection avec la droite $\mu H=0$ donne le rapport $\frac{\sigma_{\mathrm{sp}}}{\sigma_{0}}$. C'est ainsi que les valeurs du tableau suivant ont été déterminées :

\begin{tabular}{|c|c|}
\hline$T$ & $\frac{\sigma_{\mathrm{sp}}}{\sigma_{0}}$ \\
\cline { 1 - 2 } & \\
$354^{\circ} 96$ & 0,1037 \\
$331^{\circ} 90$ & $0,207 \mathrm{r}$ \\
$348^{\circ} 89$ & 02665 \\
$345^{\circ} 89$ & 0,3090 \\
$340^{\circ} 01$ & 0,3738 \\
$334^{\circ} 22$ & 0,4189 \\
$322^{\circ} 97$ & 0,4820 \\
$312^{\circ} 14$ & 0,5286 \\
& \\
\hline
\end{tabular}

Sur la figure 8, j'ai tracé, en trait plein, la courbe théorique donnant le carré de l'aimantation spontanée en fonction de la température. J'ai admis la valeur

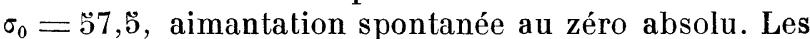
cercles sont les points expérimentaux de Weiss et Forrer. La courbe en traits interrompus représente la courbe d'aimantation spontanée obtenue avec une loi d'aimantation en tangente hyperbolique, mais sans faire intervenir les fluctuations du champ moléculaire. L'introduction des fluctuations améliore beaucoup l'accord de la théorie avec l'expérience.

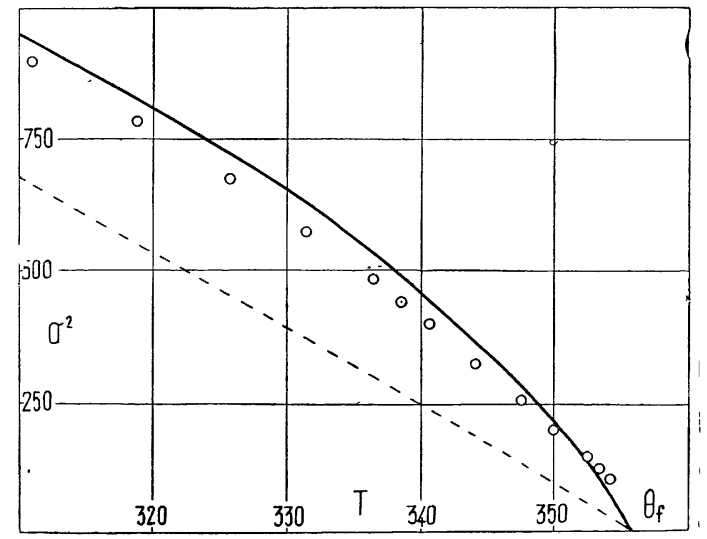

Fig. 8 .

13. Les isothermes au voisinage du pointide Curie. - Les résultats du $\$ 11$, relatifs aux aimantations faibles, montrent que la quantité $\sigma_{0}$ que nous avions supposée constante varie en réalité depuis $\sigma_{0}=77$ pour les aimantations très faibles jusqu'à $\sigma_{0}=\check{\jmath} \check{\imath}, \breve{b}$ pour la saturation.

Pour les aimantalions intermédiaires, il semble, 
priori, impossible d'utiliser les formules établies cidessus puisque la loi supposée qui relie le nombre des porteurs à l'aimantation est inconnue. La formule (̋̈) n'a un sens précis que pour un nombre fixe de porteurs dont le moment est égal à un magnéton de Bohr. J.ai montré dans le \$11 qu'elle était valable pour de petites aimantations $(\sigma<1)$, avec $\sigma_{0}=77$, et les constantes caractéristiques du champ moléculaire ayant les va- leurs suivantes : $\Theta=642^{\circ} 8, \quad n=2400, \quad V=7 \ddot{\partial} 0$. Je vais maintenant essaỵer de représenter empiriquement. les isothermes expérimentales, par la formule $\check{\partial}$, conservant les mêmes constantes du champ moléculaire mais en considérant cette fois-ci $\sigma_{0}$ et $\mu$ comme constantes d'ajustement fonction de la température. L'expérience montre que c'est possible avec une très grande précision.

Tablead VI.

\begin{tabular}{|c|c|c|c|c|c|c|c|c|}
\hline$\frac{N \mu(H+n \sigma)}{k T}$ & 372077 & $369^{\circ} 07$ & $365^{\circ} 36$ & $361^{\circ} 84$ & $358^{\circ} 03$ & $354^{\circ} 96$ & $351^{\circ} 09$ & $346^{09}$ \\
\hline $\begin{array}{l}1 \\
2 \\
3 \\
4 \\
5 \\
6 \\
7\end{array}$ & $\begin{array}{l}0,03877 \\
0,07640 \\
0,11192 \\
0,14464 \\
0,17427\end{array}$ & $\begin{array}{c}0,04296 \\
0,08439 \\
0,12303 \\
0,15810 \\
0,18928\end{array}$ & $\begin{array}{l}0,04781 \\
0,09367 \\
0,13578 \\
0,17326 \\
0,20593\end{array}$ & $\begin{array}{l}0,05326 \\
0,10377 \\
0,14946 \\
0,18925 \\
0,2232\end{array}$ & $\begin{array}{l}0,16601 \\
0,20827 \\
0,2432 \\
0,2718\end{array}$ & $\begin{array}{l}0,18148 \\
0,22000 \\
0,2606 \\
0,2890 \\
0,3120\end{array}$ & $\begin{array}{l}0,1971 \\
0,2427 \\
0,2785 \\
0,3067 \\
0,3287\end{array}$ & $\begin{array}{c}0,2739 \\
0,3093 \\
0,3359 \\
0,3561\end{array}$ \\
\hline
\end{tabular}

Un point courant d'une isotherme se détermine par la méthode indiquée au paragraphe précédent. Le tableau VI donne les valeurs de $\frac{\sigma}{\sigma_{0}}$ en fonction de $\frac{N \mu(H+n \sigma)}{k T}$ pour 8 températures différentes. Par tâtonnements, on arrive très vite à déterminer les valeurs de $\tau_{0}$ et de $\mu$ qui permettent de représenter fidèlement l'isotherme choisie. Le tableau VII donne les valeurs de $\sigma_{0}$, de $\frac{N \mu}{k T}$ et de $\mu$ (colonnes 2,3 et 4 ) qui correspondent aux valeurs de la température indiquées dans la colonne 1. Sur la figure 9, j'ai représenté par des cercles les valeurs ainsi calculées. Les croix représentent les points expérimentaux de Weiss et Forrer $(*)$.

Tableav VII.

\begin{tabular}{|c|c|c|c|}
\hline$T$ & $\sigma_{0}$ & $\frac{k T}{N^{\prime} \mu}$ & $\mu$ \\
\hline $\begin{array}{l}372^{\circ} 77 \\
369^{\circ} 07 \\
365^{\circ} 36 \\
361^{\circ} 84 \\
38^{\circ} 03 \\
354^{\circ} 96 \\
331^{\circ} 9 \\
346^{\circ} 9\end{array}$ & 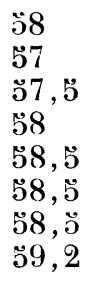 & $\begin{array}{rr}9 & 320 \\
9 & 118 \\
9 & 190 \\
9 & 230 \\
9 & 390 \\
9 & 462 \\
9 & 551 \\
10 & 050\end{array}$ & $\begin{array}{ll}7 & 690 \\
7 & 810 \\
7 & 700 \\
7 & 630 \\
7 & 450 \\
7 & 360 \\
7 & 200 \\
6 & 840\end{array}$ \\
\hline
\end{tabular}

(*) Le calcul a été fait pour une substance ayant le même champ démagnétisant que l'échantillon étudié par Weiss et Forrer.
L'examen du tableau VII montre que $\sigma_{0}$ reste très voisin de 58, pour toutes les isothermes, tandis que $\mu$ varie systématiquement. Comme $\mu$ n'est ici qu'un coefficient d'ajustement dont la signification physique n'est pas immédiate, je n'insisterai pas sur ses variations. La constante $\sigma_{0}$ n'est, elle aussi, qu'une constante d'ajustement, mais c'est, en même temps, l'aimantatation de la substance dans un champ extérieur infini.

On voit déjà à l'examen de la figure 9 , que la formule d'interpolation est bonne, puisqu'il suffit de déterminer deux constantes, c'est-à-dire un point et une tangente pour que tous les points d'une isotherme soient représentés avec précision. Mais, de plus, puisque $\sigma_{0}$ est sensiblement indépendant de la température, avec une valeur égale à la saturation absolue du nickel, on peut dire que la formule est utilisable pour des aimantations beaucoup plus fortes que celles qui ont été atteintes par Weiss et Forrer, dans les limites de température envisagées $\left(: 4 \mathrm{j}^{\circ} \mathrm{C}\right.$ à $372^{\circ} \mathrm{C}$ ). En effet, ces coïncidences ne sont pas dues au hasard. Pour résumer cette discussion, disons qu'il suffit d'une seule constante d'ajustement $\mu$, fonction de la température, pour représenter empiriquement les isothermes expérimentales. La deuxième constante $\epsilon_{0}$ est indépendante de la température : elle à, aussi bien en principe qu'en fait, la signification d'une saturation absolue.

Il est tout à fait curieux et instructif que la saturation absolue correcte du nickel soit ainsi obtenue d'après des isothermes qui correspondent à des températures supérieures au point de Curie, c'est-à-dire d'après des données qui correspondent toutes (en particulier les constantes du champ moléculaire) à des propriétés du nickel à l'état paramagnétique. Ceci 
montre bien la continuité et l'unité des états para et ferromagnétique.

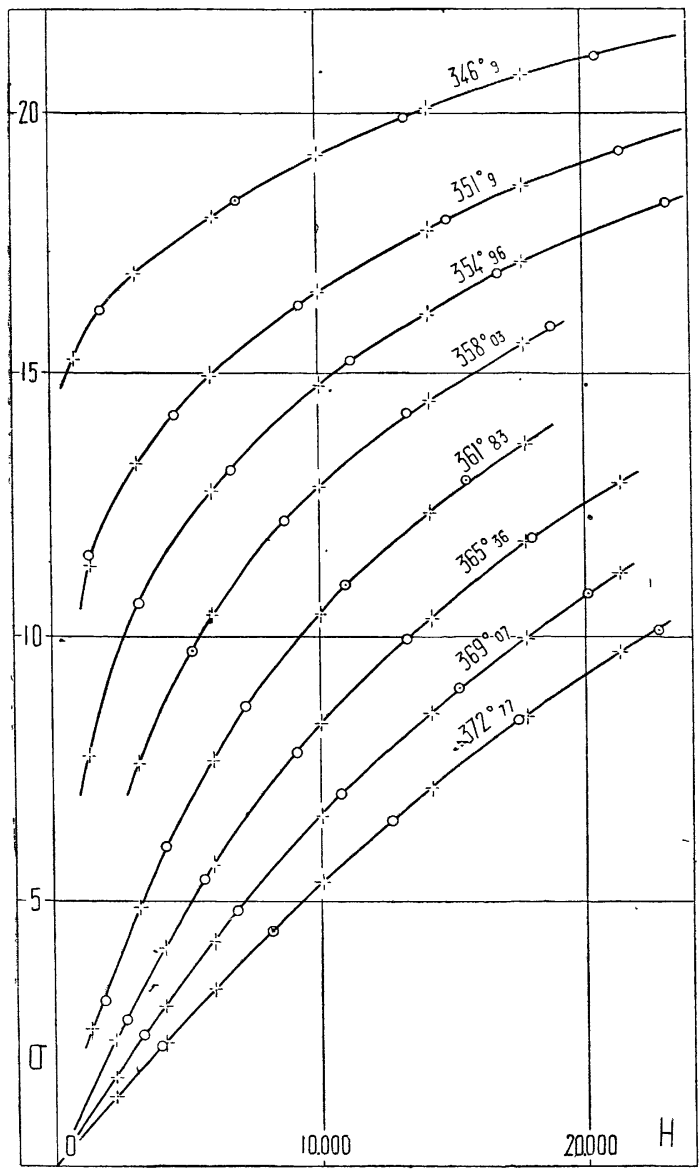

Fig. 9.

Ainsi la formule (ä), utilisée comme formule d'interpolation avec $\mu$ variable suivant la température, est correcte pour des aimantations fortes : mais le $\$ \mathbf{1 1}$ montre qu'elle n'est pas valable, tout au moins avec la mème valeur de $\sigma_{0}$, pour des aimantations faibles de quelques unités C. G.S. A une température donnée, une seule formule ne suffit pas pour repésenter empiriquement l'ensemble de la courbe $\sigma=f(\boldsymbol{H})$.

14. Calcul de la chaleur spécifique. - Il reste à montrer que les anomalies de chaleur spécifique du modèle proposé s'accordent avec l'expérience. L'énergie d'un groupe $(p, q)$ donné, de $\nu$ porteurs, est la somme de l'énergie interne $p q w$ du groupe et de l'énergie magnétique des porteurs dans le champ magnétique : - $(p-q) \mu . H$. Si on se borne à l'étude de l'aimantation spontanée, $\boldsymbol{H}=\mathbf{n} \sigma:$ l'énergie magnétique est $-\frac{1}{2} n \tau^{2}$.

Comme le nombre des groupes contenus dans $1 \mathrm{~g}$ de substance est $\frac{\sigma_{0}}{N \mu}$, l'énergie totale, par gramme, s'écrit :

$$
\frac{\sigma_{0}}{N \mu} \sum p q u \sigma-\frac{1}{2} n \sigma_{\mathrm{sp}^{2}}
$$

En tenant compte des relations :

$$
n=0,02 \frac{k \Theta}{\sigma_{0} \mu .} \quad w=\frac{2 k \Theta}{N-1},
$$

et en confondant $N$ avec $\mathrm{N}-1$, l'énergie par atomegramme s'écrit :

$$
U=2 \vee R \Theta\left[\sum \frac{p q \omega}{N^{2}}-\frac{1}{200}\left(\frac{\sigma_{\mathrm{sp}}}{\sigma_{0}}\right)^{2}\right] ;
$$

$R$ est la constante des gaz, et $v$ le nombre des porteurs par atome. L'expression entre parenthèses, soit $V$, se calculera comme nous avons calculé plus haut des quantités analogues. La chaleur spécifique atomique, en unités de Dulong et Petit $\frac{R}{2 . J}$, est :

$$
c=-4 \vee \frac{\mathrm{d} V}{\mathrm{~d} \frac{T}{\Theta}} .
$$

L'énergie $U$ dépend des constantes du champ moléculaire $n, \Theta, N$ dont les valeurs sont connues, de la température et du rapport $\nu$ du nombre des porteurs au nombre des atomes.

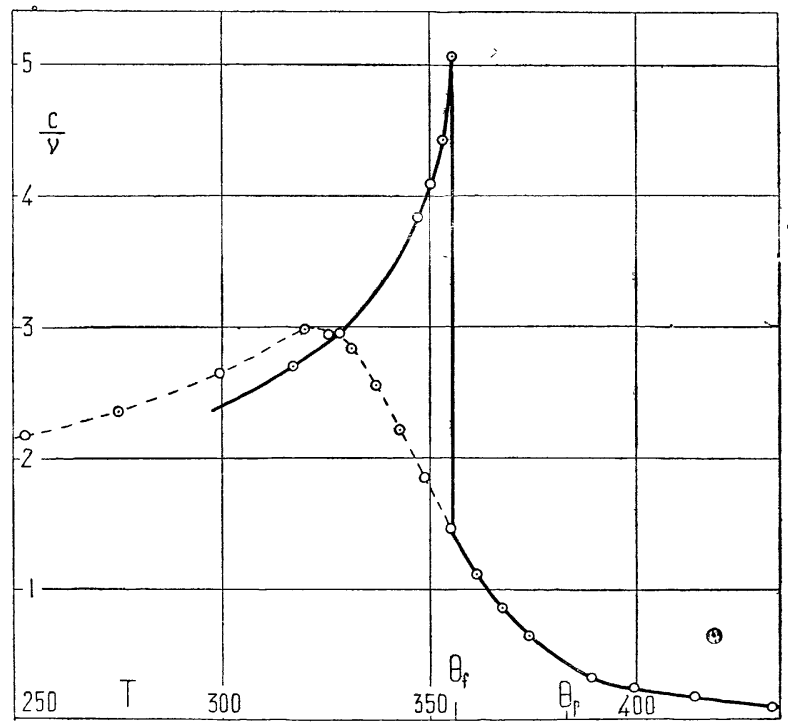

Fig. 10 .

J'ai calculé $\frac{U}{\nu}$ en fonction de $\frac{T}{\Theta}$, et j'en ai déduit, par dérivation, $\frac{c}{v}$. Les résultats font l'objet du tableau VIII.

La figure 10 représente, en trait plein, la variation de la chaleur spécifique avec la température.

Si le coefficient $u$ du champ moléculaire à longue distance était nul, il subsisterait encore une anomalie 
de chaleur spécifique représentée par la courbe pointillée. Naturellement, au-dessus du point de Curie, les deux courbes coïncident puisque les actions mutuelles à longue distance s'annulent pour une aimantation nulle.

Tableid VIII.

\begin{tabular}{|c|c|c|c|}
\hline$T$ & $\frac{c}{v}$ & $T$ & $\frac{c}{v}$ \\
\hline วัว8" 3 & 0,020 & $361^{\circ} 1$ & 1,108 \\
\hline $507^{\circ} 9$ & 0,032 & & 1,400 \\
\hline $463^{\circ} 1$ & 0,048 & $3 อ 5^{\circ}$ & 5,064 \\
\hline $432^{\circ} 5$ & 0,092 & $303^{\circ} 4$ & 4,420 \\
\hline $413^{\circ} 6$ & 0,172 & $348^{\circ} \mathrm{o}$ & 4,092 \\
\hline $399^{\circ} 2$ & 0,240 & $347^{\circ} 4$ & 3,836 \\
\hline $388^{\circ} 9$ & 0,324 & $340^{\circ} 0$ & 3,468 \\
\hline $380^{\circ} \mathrm{b}$ & 0,516 & $328^{\circ} 6$ & $2, y 52$ \\
\hline $373^{\circ} 8$ & 0,652 & $317^{\circ} 6$ & 2,700 \\
\hline $367^{\circ} 4$ & 0,856 & & \\
\hline
\end{tabular}

Le trait le plus frappant de la courbe de chaleur spécifique est la persistance, après la discontinuité, d'une anomalie de chaleur spécifique, qui disparaît ensuite graduellement. On remarque encore que la discontinuité ici égale à 3,66 y est plus grande que la discontinuité d'une substance à champ moléculaire sans fluctuations qui n'est que de $3 \nu$. Cette grande discontinuité est à rapprocher de la variation avec la température du carré de l'aimantation spontanée, au voisinage du point de Curie, plus rapide avec des fluctuations que dans le cas classique.

15. Détermination de v. - On sait que $M$. Weiss a montré (12) l'existence dans la chaleur spécifique du nickel d'un terme d'origine incertaine qu'il a appelé terme inconnu. Au-dessous du point de Curie ce terme est une fonction de la température, et parait constant au dessus. On sait aussi que la chaleur spécifique d'un nickel idéal, débarrassé de son ferromagnétisme, ne serait pas constante comme le veut la théorie classique : aux basses températures se produit la dégénérescence quantique : aux hautes températures, les oscillateurs ne sont plus harmoniques d'où le terme croissant de Born et de Brody. Pour ces différentes raisons, il vaut mieux comparer la théorie à l'expérience dans un domaine de température aussi réduit que possible pour que les termes perturbateurs varient peu : c'est pourquoi je comparerai d'abord les discontinuités. La théorie donne 3,66 v et l'expérience donne $2,10 ろ$ (ce nombre est obtenu après la réduction à volume constant et après la correction de dégénérescence quantique). La comparaison donne $\nu=0,575$.

Cette méthołe semble brutale : la discontinuité obtenue par Mme Lapp (3) est le résultat d'une extrapolation, car le nickel utilisé dans ses expériences ne présentait pas de discontinuité franche : la chute de la chaleur spécifique était étalée sur un intervalle de $\breve{J}^{\circ}$ à $6^{\circ}$, ce qui était vraisemblablement dû à un mélange de plusieurs variétés de nickel avec des points de Curie légèrement différents. Mais l'extrapolation indispensable a été faite par Mme Lapp dans l'hypothèse où il ne subsistait aucune anomalie au-dessus du point de Curie. Etant données les difficultés qu'il y aurait à restituer la discontinuité correcte, j'ai jugé préférable de comparer les chaleurs spécifiques de deux points, situés de part et d'autre du point de Curie, à une distance assez grande pour que les erreurs provenant de l'étalement du point de Curie soient très atténuées, mais pas trop grande cependant, pour que les termes perturbateurs n'aient pas varié sensiblement.

J'ai choisi les températures de $35^{\circ}$ et de $362^{\circ}$; les résultats sont condensés dans le tableau suivant:

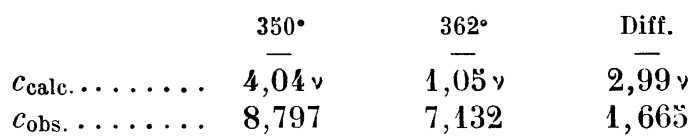

En égalant les deux valeurs données dans la dernière colonne, on obtient :

$$
\nu=0,, 356 .
$$

Cette valeur est un peu plus faible que celle qui avait été calculée d'après la discontinuité. Mme Lapp a évalué l'erreur relative maximum sur la chaleur spécifique totale à \pm 2 pour 100 . L'erreur absolue vers le point de Curie serait donc de $\pm 0,16$. J'admettrai que l'erreur absolue sur la différence de deux chaleurs spéci fiques est aussi de $\pm 0,16$, en supposant une compensation partielle des erreurs. On en déduit :

$$
\nu=0,556 \pm 0,056 \text {. }
$$

Il est probable que le nombre $\nu$, qui intervient dans la chaleur spécifique, est relatif aux porteurs effectivement orientés à la saturation. L'hypothèse $S=\frac{1}{2}$ fournit à basse température 0,607 porteur par atome (§ 1). Ce nombre s'accorde avec la valeur de y calculée plus haut.

\section{Corrections de mécanique quantique.}

16. Le ferromagnétisme traité par la mécanique quantique. - Les calculs que j'ai développés dans la première partie de cet exposé ont un caractère semi-classique; ils ont l'inconvénient de négliger l'origine du champ moléculaire qui ne peut être correctement interprété que par la mécanique quantique. Certes, les fluctuations sont ụn phénomène général qui intervient aussi bien en statistique quantique qu'en statistique classique, mais il faut préciser.

J'utiliserai le modèle quantique de Heisenberg, avec le mode de calcul donné par Pauli dans son rapport au

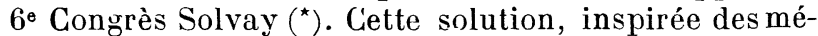

(*) Le Magnétisme. Rapports et discussions du $6^{\circ}$ Congrès Solvay (Gauthier-Villars), Paris, 1932. 
thodes de Dirac et de Slater, se présente sous une forme favorable pour traiter le problème des fluctuations d'une manière analogue à celle que j'ai employée plus haut. Je ne reproduirai pas les calculs et je me contenterai de citer ce qui est strictement indispensable à mon objet.

Soit un groupe de $N$ porteurs, chacun dans l'état $S$, avec un seulélectron; la fonction propre non perturbée de chaque porteur est connue. On introduit les actions mutuelles sous la forme d'une perturbation, et les fonctions propres perturbées du groupe sont développées au moyen des fonctions propres non perturbées, en négligeant en particulier les états hétéropolaires.

Soit $m$, le nombre quantique qui représente $\left(^{*}\right)$, pour un état possible du système, la composante du moment angulaire résultant suivant la direction du champ magnétique. Le principe d'exclusion de Pauli limite à $\left.r=C_{N}^{\frac{N}{2}+m}{ }^{* * *}\right)$, le nombre de solutions distinctes possédant un no mbre quantique $m$. Pour écrire sous une forme rigoureuse la somme d'état $Z$, d'où l'on déduit l'aimantation totale du système par la formule :

$$
\sigma=k T \frac{\partial}{\partial \boldsymbol{H}} \log Z
$$

il faut connaître l'énergie correspondant à chaque solution, c'est-à-dire résoudre une équation du rième degré. Devant l'impossibilité d'arriver rigoureusement à ce résultat, on calcule simplement la somme des énergies propres des $r$ solutions, ce qui est facile, et on utilise les remarques suivantes:

La résultante $S$ du moment angulaire, dans un état d'énergie $E$, est, en l'absence de champ magnélique extérieur, dirigée suivant une direction quelconque de l'espace : mais si on fait agir un champ magnétique, la composante de $S$ suivant la direction du champ magnétique peut prendre seulement les valeurs $S, S-1, S-2$, ...-S. Naturellement, l'énergie de chacun de ces états quantifiés reste égale à $E$. Réciproquement, les états qui correspondent à une composante $m$ du moment angulaire suivant la direction du champ magnétique proviennent de différents états dont les moments angulaires résultants et les énergies sont différents.

On peut alors calculer l'énergie moyenne des états dont le moment angulaire résultant est $s$ :

$$
\bar{E}_{s}=H_{i}-\frac{N^{\top}(N-4)+4 s(s+1)}{2 N(N-1)} \sum_{i<k} H_{i k} ;
$$

$H_{i}$ représente l'énergie non perturbée et $H_{i k}$ l'intégrale de permutation entre deux atomes de rang $i$ et $k$.

$$
\begin{aligned}
& \text { En fonction de l'unité } \frac{h}{2 \pi} \text {. } \\
& (\star \star) \text { Je pose comme d'habitude : } \\
& \qquad c_{\checkmark}^{\frac{x}{2}+m}-\frac{N !}{\left(\frac{N}{2}+m\right) !\left(\frac{N}{2}-m\right) !}
\end{aligned}
$$

Dans les calculs ultérieurs, on remplace les différents états de résultante $s$, possédant des énergies différentes, par un seul état d'énergie $\bar{E}_{s}$, affecté du poids statistique :

$$
f(s)=C_{N}^{\frac{N}{2}+s} \frac{2 s+1}{\frac{N}{2}+s+1}
$$

Ces formules sont valables quelles que soientles valeurs $\boldsymbol{H}_{i k}$ des intégrales de permutation en fonction des $\boldsymbol{i}$ et des $k$. Mais leur utilisation à écrire une somme d'état $Z$, ne fournit qu'une solution approchée d'autant plus exacte que les énergies des différents états de résultante $s$ s'écartent moins de l'énergie moyenne $E_{s}$. Cet écart serait minimum si les énergies d'échange étaient uniformément réparties sur tous les couples possibles de deux atomes pris parmi les $V$ du groupe. C'est-àdire si tous les atomes jouaient le mème rôle les uns par rapport aux autres.

Le traitement de ce problème de mécanique quantique oblige donc pratiquement, par suite de l'insuffisance des solutions mathématiques, à supposer implicitement que les $H_{i k}$ sont indépendants des $i$ et des $k$. Autrement dit, on considère comme équivalents tous les problèmes cù la valeur moyenne des $\boldsymbol{H}_{i k}$ est la même. Ces conventions sont identiques à celles que j'ai adoptées pour le modèle semi-classique utilisé dans ce mémoire.

On étudie ainsi les lois d'aimantation d'un groupe de $V$ atomes dont les liaisons sont identiques. Mais tandis que dans le modèle semi-classique,j'avais adopté une valeur finie de $N$, pour provoquer arlificiellement des fluctuations sous une forme mathématiquement accessible, dans le modèle quantique on fait tendre $N$ vers l'infini, l'effet de fluctuation est ainsi éliminé et on retrouve donc la théorie de Weiss.

Pour rendre compte des fluctuations, en évitant l'introduction implicite d'intégrales de permutation égales quelque soit la distance des atomes, il faudrait, par exemple, perfectionner le calcul en tenant compte du carré moyen de l'écart $\boldsymbol{E}_{s}-\overline{\boldsymbol{E}_{s}}$. Cependant des calculs parallèles effectués sur le modèle semi-classique montrent que l'approximation ainsi obtenue est encore très mauvaise au point de Curie.

17. Comparaison du modèle semi-classique et du modèle quantique. - Puisque le modèle semiclassique, avec $N$ fini, fournit des résultats qui cadrent fort bien avec l'expérience, l'adoption en mécanique ondulatoire, d'un artifice de calcul analogue doit être fructueuse

Quoiqu'il en soit, les fonctions de partition, $Z$ du modèle ondulatoire (*) et $Z^{\prime}$ du modèle semi-classique,

(*) Cette formule est directement déduite des équalions ว2 et วั4 du rapport de Pauli déjà cité, avec des changements de notation insignifiants et les approximations légitimes lorsque $N$ est assez grand pour négliger $N$ devant $N^{2}$. 
s'écrivent au mème ordre d'approximation près, en posant $x=\frac{\mu H}{k T}$ :

$$
\begin{aligned}
Z=\sum_{p=\frac{N}{2}}^{N} C_{N}^{p}\left(1-\frac{q}{p+1}\right) \mathbf{e}^{\frac{p q A}{k T}} & \\
& {\left[\mathbf{e}^{(p-q) \alpha}+\mathbf{e}^{(p-q-q) \alpha}+\ldots+\mathbf{e}^{(q-p) \alpha}\right] }
\end{aligned}
$$$$
Z^{\prime}=\sum_{p=\frac{N}{\underline{\nu}}}^{N} C_{N}^{p} \mathrm{e}^{\frac{p q A^{\prime}}{k T}}\left[\mathbf{\theta}(p-q) \alpha+\mathbf{e}^{(q-p) \propto]}\right.
$$

avec

$$
p+q=N
$$

$\boldsymbol{A}$ se calcule, en principe, au moyen des intégrales de permutation :

$$
A=-\frac{2}{N^{2}} \sum_{i<k} I_{i k}
$$

et $A^{\prime}$ au moyen des énergies d'action mutuelle définies au début de cet article $\left(^{\star}\right)$ :

$$
A^{\prime}=-u=-\frac{2}{N^{2}} \sum_{i<k} w_{\imath k}
$$

Ces fonctions de partition $Z$ et $Z^{\prime}$ sont rigoureuses si les $H_{i k}$ et les $w_{i k}$ sont tous égaux. Elles ne sont qu'approchées dans le cas contraire.

Aux températures élevées, pour des aimantations faibles $Z$ et $\boldsymbol{Z}^{\prime}$ redonnent asymptotiquement la même loi d'aimantation en tangente hyperbolique. C'est aux basses températures, au voisinage de la saturation, qu'elles différent le plus.

Dans ce dernier cas, $\frac{q}{p+1}$ est petit et les coefficients des exponentielles sont pratiquement identiques dans $Z$ et dans $Z^{\prime}$. La seule différence fondamentale, entre les deux modèles, est la suivante : dans le modèle semi-classique, j’ai supposé que la composante du moment magnétique suivant la direction du champ magnétique ne prenait que les valeurs $(p-q) \mu$. et $-(p-q) \mu$, tandis que la mécanique quantique nous apprend qu'elle peut prendre en réalité la série de valeurs $(p-q) \mu,(p-q-2) \mu, \ldots-(p-q) \mu$.

Comme le montre la comparaison du calcul à l'expérience, le modèle semi-classique suffit au voisinage du point de Curie, mais il est complètement en défaut devant les lois expérimentales d'approche à la saturation.

18. Corrections à apporter aux calculs semiclassiques. - Plutôt que de recommencer avec le modèle quantique des calculs longs et fastidieux, j'ai

(*) On prend égale à $w$ l'énergie potentielle de deux atomes $i$ et $k$ antiparallèles et zéro comme énergie potentielle de deux atomes parallèles (cf. 10, p. 8). préféré retoucher le modèle classique, ce qui peut se faire rigoureusement au voisinage de la saturation.

Près de la saturation $\sigma_{0}$. l'influence des fluctuations est devenue négligeable, et l'aimantation du groupe de $I^{\prime}$ atomes s'écrit d'après la loi en tangente hyperbolique :

$$
S=N \mu\left(1-2 \mathrm{e}^{-\frac{2 \theta_{\mu}}{T}}\right)
$$

Cetle aimantation provient d'états du groupe parallèles au champ magnétique et d'états antiparallèles, mais la probabilité de ces derniers est absolument insignifiante, de sorte que $S$ représente aussi la résultante moyenne du groupe, à laquelle on peut appliquer les résultats du calcul quantique. La composante de $S$ dans la direction du champ magnétique, est quantifiée avec un grand nombre de valeurs possibles équidistantes. La saturation correcte s'obtient donc en appliquant la loi de Langevin à des porteurs de moment $s$; et l'aimantation provenant d'un groupe de $N$ porteurs s'écrit :

$$
\begin{aligned}
\sigma & =S\left(\operatorname{coth} a-\frac{1}{a}\right), \\
\text { en posant : } & a=\frac{S\left(H_{m}+H\right)}{k T}
\end{aligned}
$$

$H_{m}$ est égal au champ moléculaire à longue distance : $H$ est le champ magnétique extérieur. La comparaison des équations (16) et (17) donne, en négligeantles termes du second ordre, en confondant la tangente hyperbolique avec 1 , et en remplaçant $S$ par $N \mu$ dans le terme correctif $\left(^{*}\right)$ :

$$
\sigma=\sigma_{0}\left[1-\frac{k T}{N \mu\left(H+H_{m}\right)}-2 \mathrm{e}^{-\frac{2 \theta_{p}}{T}}\right] .
$$

Cette équation donne la loi d'approche à la saturation en fonction de la température et du champ magnétique. Toutes les constantes qui figurent dans cette équation ont été déterminées dans les paragraphes (5) à (7), par l'étude du nickel paramagnétique. J'adopterai ainsi :

$$
{ }_{0}{ }_{p}=655^{\circ}, \quad N=750, \quad H_{m}=2400 \sigma_{0} .
$$

Une discussion antérieure a permis d'adopter comme moment du porteur le magnéton de Bohr soit $5564 \mathrm{C}$. G. S. Enfin, l'étude des isothermes voisines du point de Curie donne pour $\sigma_{0}$ une valeur voisine de 58. La précision avec laquelle cette valeur est connue n'est naturellement pas suffisante pour étudier de près les lois d'approche à la saturation. J'ai donc adopté la valeur de $\tau_{0}$ qui donnait l'accord le meilleur dans ces conditions soit : $\sigma_{0}=58,94$.

19. Loi d'approche en fonction de la température $(13)$. - Je suppose le champ extérieur nul. J'ai

*) L'étude des valeurs numériques données plus loin montre que toutes ces approximations sont justifiées. 
calculé, à différentes températures, les valeurs de $\sigma_{0} \frac{k T}{\Gamma_{\mu} H_{m}}$ de $2 \sigma_{0} \mathrm{e}^{-\frac{2 \theta_{p}}{T}}$ et de $\sigma(\operatorname{colonnes} 1,2,3$ et $4 \mathrm{du}$ tableau IX). La dernière colonne donne les valeurs expérimentales de Weiss et Forrer (ㄹ, p. 322). Les valeurs calculées coïncident avec les valeurs expérimentales à moins de sept dix-millièmes près. Sur la figure 11 j'ai

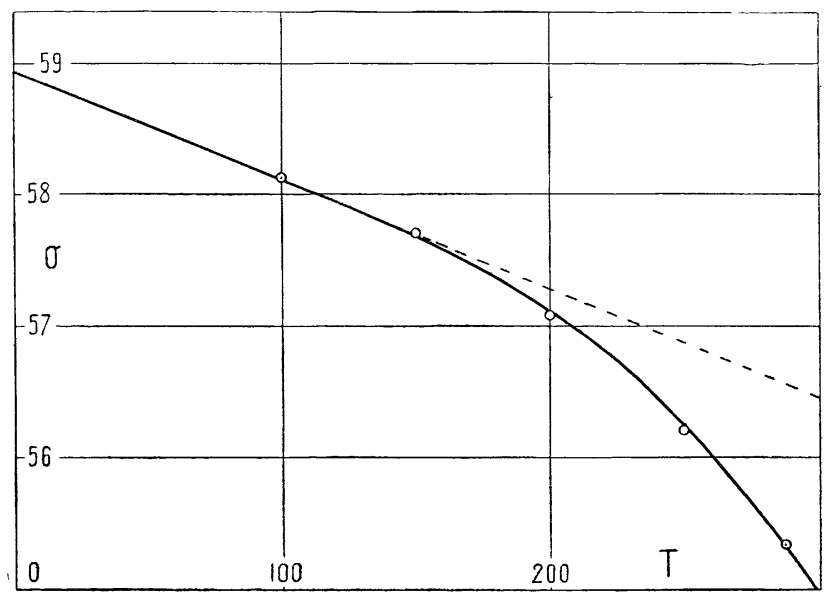

Fig. 11 .

représenté en trait plein la courbe calculée. Les cercles sont les points expérimentaux. La droite pointillée est la tangente à l'origine. La loi d'approche en $T$ n'est pas valable au dessus de $100^{\circ} \mathrm{K}$. Cette loi d'approche en $T$ tient au modèle employé, relatif à un nombre fini d'atomes. Pour un nombre infini, on sait que la Ioi d'approche quantique rigoureuse est en $T^{\overline{3}}$. Ici, par suite de la méthode d'approximation employée, le terme en $T^{\overline{3}}$ est rẻmplacé par deux termes, l'un en $T$ et l'autre exponentiel.

Tableau IX.

\begin{tabular}{|c|c|c|c|c|}
\hline$T^{\circ}$ & $\frac{\sigma_{0} k T}{\bar{N} \mu H_{m}}$ & $2 \sigma_{0} e^{-\frac{2 \eta_{p}}{T}}$ & $\sigma$ calc. & $\sigma$ obs. \\
\hline $100^{\circ}$ & 0,83 & 0,00 & 58,11 & วั8,13 \\
\hline $150^{\circ}$ & 1,24 & 0,02 & $\check{3} 7,68$ & 57,71 \\
\hline $200^{\circ}$ & 1,65 & 0,17 & 57.12 & 37,08 \\
\hline $250^{\circ}$ & 2,07 & 0,63 & $\ddot{6} 6,24$ & $\ddot{2} 6,20$ \\
\hline$\underline{488^{\circ}}$ & 2,38 & $1,2 \circlearrowright$ & ธั5้.31 & ๖ัว, $3 \mathbf{4}$ \\
\hline
\end{tabular}

L'accord inespéré de la théorie avec l'expérience montre que le modèle utilisé suffit pour des températures supérieures à $100^{\circ} \mathrm{K}$. Au-dessous, il n'y a pas encore de données expérimentales pour le juger.
20. Loi d'approche en fonction du champ magnétique (13). - A la saturation, le champ moléculaire à longue distance est de $\mathbf{1 3 9 . 0 0 0}$ gauss. Lorsque le champ extérieur est comparativement petit, on peut écrire :

$$
\sigma=\sigma_{0}\left[1-\frac{h T}{N \mu H_{m}}\left(1-\frac{H}{H_{m}}\right)\right]
$$

ou :

$$
\sigma=\sigma_{T}(1+b H)
$$

en désignant par $\sigma_{T}$, la saturation relative à la température $T$, dans un champ nul. On a sensiblement :

$$
b=\frac{l \cdot T}{N H_{0} H_{m^{2}}}=1,02 T \times 10^{-9}
$$

Ce terme correspond exactement à l'aimantation parasite de Weiss et Forrer. La valeur numérique de $b$ calculée à $288^{\circ} \mathrm{K}$ est $0,29 \times 10^{-b}$, alors que les mèmes auteurs trouvent $0,22 \times 10^{-6}$. Si l'on remarque que dans un champ de 10.000 gauss, $b H$ ne représente pas trois pour mille de l'aimantation totale, l'accord parait satisfaisant, étant donné la difficulté des expériences $\left(^{*}\right)$.

\section{Conclusions.}

21. Résultats de l'étude de l'équation d'état magnétique du nickel. - Les résultats acquis à l'issue de cette étude générale du nickel se classent en deux catégories: les uns, relatifs aux fluctuations du champ moléculaire, sont d'ordre purement formel let n'intéressent que la portion du réseau d'isothermes magnétiques voisine du point de Curie : les autres, d'ordre plus général, nous renseignent sur la nature du porteur élémentaire du nickel.

\section{Les fluctuations du champ moléculaire. -} Les arguments en faveur de cette hypothèse sont les suivants :

$1^{\circ}$ Les relations avec la température de la susceptibilifé du nickel, dans l'état paramagnétique, s'interprètent quantitativement. D'ailleurs, aucune autre interprétation n'a été donnée.

$2^{\circ}$ L'aimantation spontanée calculée ne présente plus avec les valeurs observées que de légers écarts.

$3^{\circ}$ Les anomalies de chaleur spécifique sont correctement représentées : les conclusions qu'on déduit de leur étude s'accordent avec celles que fournit l'étude des propriétés proprement magnétiques.

( ) On sait que la loi d'approche complète à la saturation à la température $T$ est le la forme :

$$
\sigma=\sigma_{\imath}\left(1-\frac{a}{H}+b H\right)
$$

$b$ est à peu près constant pour tous les échantillons mais $a$ varie dans de larges limites. Le terme $a$ est probablement à rattacher aux actions cristallines et intercristallines. Toutefois M. Forrer (1'́) en a donné une interprétation différente et séduisante. 
$4^{\circ} \mathrm{La}$ théorie fournit une formule empirique, qui ne contient que deux constantes arbitraires et qui permet de représenter avec précision les isothermes voisines du point de Curie.

$\breve{\zeta}^{\circ}$ Certaines corrections de mécanique quantique qu'il est nécessaire d'apporter aux calculs, au voisinage de la saturation, permettent de rendre compte quantitativement de la loi d'approche à la saturation et donnent correctement le terme d'aimantation parasite. Les données utilisées dans ces derniers calculs sont toutes empruntées à l'étude du nickel paramagnétique.

L'ensemble de ces arguments prouve, d'une manière incontestable, le ròle important des fluctuations du champ moléculaire.

Il faut remarquer que l'hypothèse des fluctuations du champ moléculaire ne constitue vraiment une hypothèse nouvelle que si l'on se tient strictement au point de vue formel du champ moléculaire de Weiss. Si l'on part au contraire, d'après Heisenberg, des actions d'échange, origine du ferromagnétisme, c'est en première approximation seulement qu'on obtient le champ moléculaire de Weiss et ce sont les modifications obtenues en poussant plus loin les approximations, que, par un langage qui me semble commode, j'ai attribuées aux fluctuations de ce champ moléculaire. Je n'ai donc pas introduit d'hypothèses nouvelles, mais seulement quelques procédés de calcul destinés à préciser les résultats fournis par les mêmes hypothèses fondamentales.

23. La nature du porteur élémentaire du nickel. - L'effet gyromagnétique montre que le moment orbital ne joue aucun rôle dans le ferromagnétisme : les orbites sont bloquées et le moment n'est dù qu'au spin.

Les calculs développés ci-dessus, ne s'appliquent qu'au cas où le moment angulaire résultant d'un porteur est égal à $\frac{1}{2}$ : par exemple, quand il n'y a qu'un seul électron par porteur. Il est presque impossible d'effectuer les calculs correspondants lorsque $S$ a la valeur 1 ou une valeur supérieure. Lorsqu'on se tient à l'hypothèse $S=\frac{1}{2}$, pour interpréter à la fois la saturation $\sigma_{o}$ et la constante de Curie $C$, il faut faire varier le nombre des porteurs. Cette variation correspond-elle à la réalité ou n'est-elle qu'une apparence due à l'emploi d'une valeur incorrecte pour $S$ ?

En faveur d'une variation réelle du nombre des porteurs et de la valeur $S=\frac{1}{2}$, on peut rassembler les arguments suivants :

$1^{\circ}$ On calcule ainsi correctement la discontinuité de la chaleur spécifique et la courbure initiale des isothermes au voisinage du point de Curie.

2' L'expérience montre qu'au voisinage du point de Curie, à une température clonnée, $\frac{1}{\%}$ se développe en fonction de $H^{2}$ sous la forme suivante (voir le $\S 11$ ):

$$
\frac{1}{\chi}=a+b H^{2}+c H^{4}+\ldots
$$

$a, b, c$, étant tous les trois positifs. Il ne semble pas possible d'obtenir un développement de ce genre, par une loi d'aimantation relative à un nombre invariable de porteurs : on obtient des coefficients $a$ et $b$ posilifs mais un coefficient $c$ négatif. Par exemple, le calcul est aisé à faire pour une substance paramagnétique dépour vue de champ moléculaire. Au contraire, si le nombre des porteurs décroit quand l'aimantation croit,un coefficient $c$ positif peut se justifier.

En résumé, seule l'hypothèse d'un nombre variable avec l'aimantation, de porteurs à spin résultant : $S=\frac{1}{2}$, paraît compatible avec les faitş expérimentaux

Manuscrit reçu le 17 janvier 1934.

\section{BIBLIOGRAPHIE}

1. Weiss et Forrer, Ann. Physique, 1926, 5, p. 153.

2. Weiss et Forrer, Ann. Physique, 1929, 12, p. 279.

3. Weiss, Piccard et Carrard, Arch. Sc. phys. et nat., 1917, 42, p. 373 et 1917, 43, pp. 22, 113 et 199. Lapp (1Me) Ann. de Physique, 1929,12, p. 442 .

4. IVeiss, J. Phys., 1907, 6, p. 666.

5. Lavgevix, Ann. de Chim. et de Phys., 1903̈, 4, p. 70.

6. Stoner, Phil. Mag., 1930, 10, p. 27 .
7. Levz, Physik. Z., 1920, 21, p. 613.

8. Trler, /'hl. Mag., 1930, 9, p. 1036 et 1931, 11, p. 596

9. Wolp, Z. Pliysık, 1931, 70, p. 519.

10. NÉEL, Ann. Phystque, 1932, 17, p. 5.

11. NékL, C. R., 1933, 197, p. 1195.

12. Wriss, C.R., 1928, 187, p. 12.

13. NÉEL, $C . R ., 1933,197$, p. 1310

14. Forrer, J. Phys., 1929, 10, p. 247. 